\title{
Optimization routine for identification of model parameters in soil plasticity
}

\author{
Hans Mattsson ${ }^{1} *$, Marek Klisinski ${ }^{1}$ and Kennet Axelsson ${ }^{2}$ \\ ${ }^{1}$ Department of Civil and Mining Engineering, Luleå University of Technology, SE-97187 Luleå, Sweden \\ ${ }^{2}$ Department of Civil Engineering, School of Engineering, Jönköping University, P.O. Box 1026, SE-55111 Jönköping, Sweden
}

\begin{abstract}
SUMMARY
The paper presents an optimization routine especially developed for the identification of model parameters in soil plasticity on the basis of different soil tests. Main focus is put on the mathematical aspects and the experience from application of this optimization routine. Mathematically, for the optimization, an objective function and a search strategy are needed. Some alternative expressions for the objective function are formulated. They capture the overall soil behaviour and can be used in a simultaneous optimization against several laboratory tests. Two different search strategies, Rosenbrock's method and the Simplex method, both belonging to the category of direct search methods, are utilized in the routine. Direct search methods have generally proved to be reliable and their relative simplicity make them quite easy to program into workable codes. The Rosenbrock and simplex methods are modified to make the search strategies as efficient and user-friendly as possible for the type of optimization problem addressed here. Since these search strategies are of a heuristic nature, which makes it difficult (or even impossible) to analyse their performance in a theoretical way, representative optimization examples against both simulated experimental results as well as performed triaxial tests are presented to show the efficiency of the optimization routine. From these examples, it has been concluded that the optimization routine is able to locate a minimum with a good accuracy, fast enough to be a very useful tool for identification of model parameters in soil plasticity. Copyright (C) 2001 John Wiley \& Sons, Ltd.
\end{abstract}

KEY WORDS: optimization; constitutive models; soil plasticity; parameter identification

\section{INTRODUCTION}

Important issues in soil modelling are not only the proper choice of a constitutive model but also the identification of parameters of the chosen model. An organized way to determine model parameters is to utilize an optimization routine and base the identification on a number of available and appropriate laboratory tests. Since high-speed digital computers made implementa-

\footnotetext{
* Correspondence to: H. Mattsson, Department of Civil and Mining Engineering, Luleå University of Technology, SE-97187 Luleå, Sweden
}

Contract/grant sponsor: TFR (Swedish Research Council for Engineering Science)

Contract/grant sponsor: SBUF (the Development Fund of the Swedish Construction Industry)

Contract/grant sponsor: Swedish Building Research Council (BFR) 
tion possible, a large number of different optimization methods have been proposed, see e.g. Reference [1]. However, the performance of these optimization methods is highly dependent on the type of problem to which they are applied and most methods seem to have been investigated for a limited number of test functions only, often such that are difficult to minimise in a mathematical sense. There is, in fact, a lack of papers discussing experiences with different optimization strategies in the field of soil plasticity.

In this paper, the mathematical aspects and the experience of an optimization routine, developed for the specific purpose of identification of model parameters in soil plasticity, are focused on. The optimization routine is included as a part of a Constitutive Driver containing a library of constitutive soil models based on the flow theory of plasticity, Mattsson et al. [2]. The constitutive equations that form the mathematical basis in this Constitutive Driver are formulated as incremental tangential relations under mixed control both for drained and undrained behaviour, see References [3,4]. In the Constitutive Driver, the choice of control variables is restricted to principal stress components and/or principal strain components, i.e. no shear components are included. This implies that only such laboratory or field tests progressing under the described type of control can be simulated in the Constitutive Driver. This is the case for both conventional triaxial tests and true triaxial tests, when the stress and strain state are assumed to be homogeneous, i.e. no boundary effects are taken into account.

The mathematical procedure of optimization basically consists of two parts, the formulation of an objective function measuring the difference between theoretical and experimental results and the selection of an optimization strategy enabling the search for the minimum of this function. The mathematical strategy of the optimization routine applied here is essentially a modified and extended version of the work by Klisinski [5]. Some alternative expressions for the objective function are proposed. These are particularly appropriate in connection with the mixed control concept and simultaneous optimization against several laboratory tests. Two different search strategies, one based on Rosenbrock's method [6] and the other based on the simplex method by Nelder and Mead [7], are included in the optimization routine. Both methods belong to the category of direct search methods, and have been chosen because only objective function values are needed to perform the search, a fact leading to both generality and simplicity.

\section{MATHEMATICAL PROCEDURE}

In the optimization problem discussed here, parameters of the selected constitutive model play the role of optimization variables. Up to now, the progress in the field of constitutive soil modelling has not reached the point where it could be presumed that a selected constitutive model, with the same set of model parameters, is able to qualitatively describe all different stress/strain paths obtained in laboratory experiments on a certain soil, i.e. the values of the evaluated model parameters might, to some extent, be dependent on the type of laboratory experiment performed. An obvious way to handle this problem is to evaluate model parameters from laboratory tests with similar stress/strain paths as in the boundary value problem under inspection. This is not always possible in practise, because of the problem to anticipate the stress/strain paths in advance, before e.g. a FEM-simulation has been performed, and the fact that it is only a small portion of all 'possible' stress/strain paths that can be simulated in laboratory. However, quite generally, it seems to be advantageous to let many (qualitatively different) 
laboratory experiments form a basis for the optimization, and in that way obtain more reliable model parameters.

In the optimization process, the difference between the experimental result and the theoretical prediction for each laboratory test is measured by a norm value, here referred to as the individual norm. The individual norms of the tests form an objective function $F(\mathbf{x})$ which then gives a scalar measure of the error between the experimental observations and the model predictions. From mathematical point of view, the optimization problem involves the minimization of the objective function

$$
F(\mathbf{x}) \rightarrow \min
$$

where $\mathbf{x}$ is a vector containing the optimization variables (here model parameters) with the bound constraints

$$
\mathbf{x}_{l} \leqslant \mathbf{x} \leqslant \mathbf{x}_{\mathrm{u}}
$$

where $\mathbf{x}_{l}$ and $\mathbf{x}_{\mathbf{u}}$ are, respectively, the lower and upper bounds of $\mathbf{x}$. These bounds are introduced in order to prevent evaluation of the objective function for unreasonable states during the search iteration. In addition, as shown below, these bounds will simplify the input to the search algorithms.

The solution of the optimization problem is a vector $\mathbf{x}_{0}$ which for any $\mathbf{x}_{l} \leqslant \mathbf{x} \leqslant \mathbf{x}_{\mathrm{u}}$ satisfies the condition

$$
F\left(\mathbf{x}_{0}\right) \leqslant F(\mathbf{x})
$$

of a global minimum. However, most optimization routines are only capable of searching for a local minimum. This is also true for the direct search methods discussed here. In the general case, there is no way to check if the local minimum obtained is also the global one. A possibility to handle the problem is to start the search from different initial positions and, if the local minima become the same, it is most probably also the global minimum.

\section{THE OBJECTIVE FUNCTION}

In the formulation of an objective function, it is essential to include the stress/strain behaviour at every point in each test involved in the optimization. By that, the overall behaviour of the soil is captured.

As a first step, an expression for the individual norm has to be established. To obtain a general formulation of the individual norm, it is favourable to make it independent of the type of constitutive control (stress control, strain control or mixed control) and only let it be a function of independent variables. In optimization problems of soil plasticity such independent variables are in a natural way represented by the principal total stresses $s_{1}, s_{2}$ and $s_{3}$, the principal strains $\varepsilon_{1}, \varepsilon_{2}$ and $\varepsilon_{3}$ as well as the pore pressure $u$. Since these variables are known only in discrete points, both for the experimental result and the computed theoretical result, the individual norm will be based on Euclidean measures between such points. The Euclidean distance between an experimental point and a theoretical point is

$$
d=\left[\frac{1}{s_{0}^{2}} \sum_{i=1}^{3}\left(s_{i}^{\text {exp }}-s_{i}^{\text {theor }}\right)^{2}+\frac{1}{\varepsilon_{0}^{2}} \sum_{i=1}^{3}\left(\varepsilon_{i}^{\text {exp }}-\varepsilon_{i}^{\text {theor }}\right)^{2}+\frac{1}{u_{0}^{2}}\left(u^{\exp }-u^{\text {theor }}\right)^{2}\right]^{1 / 2}
$$


where $s_{0}, \varepsilon_{0}$ and $u_{0}$ are scaling factors necessary for the comparison of stresses and strains. The scaling factors are chosen as the maximum absolute value of all the total stresses, strains and pore pressures, respectively, in the discrete points involved in the computation of the individual norm

$$
\begin{aligned}
s_{0} & =\max \left(\left|s_{1}\right|,\left|s_{2}\right|,\left|s_{3}\right|\right) \\
\varepsilon_{0} & =\max \left(\left|\varepsilon_{1}\right|,\left|\varepsilon_{2}\right|,\left|\varepsilon_{3}\right|\right) \\
u_{0} & =\max |u|
\end{aligned}
$$

giving the inequalities

$$
\begin{aligned}
& -1 \leqslant \frac{s_{1}}{s_{0}}, \frac{s_{2}}{s_{0}}, \frac{s_{3}}{s_{0}} \leqslant 1 \\
& -1 \leqslant \frac{\varepsilon_{1}}{\varepsilon_{0}}, \frac{\varepsilon_{2}}{\varepsilon_{0}}, \frac{\varepsilon_{3}}{\varepsilon_{0}} \leqslant 1 \\
& -1 \leqslant \frac{u}{u_{0}} \leqslant 1
\end{aligned}
$$

However, under drained conditions, the pore pressure $u$ is constant and its contribution can be removed from Equation (4). Observe that, for tests where not all the experimental components in Equation (4) are determined, the space has to be reduced to the space composed by the known quantities.

The minimum distance $d_{\min }$, between experimental points and the prediction curve, for each experimental point included in the test, is searched for. It is important to observe that there is no need to compute $d_{\min }$ for the first experimental point, since the same initial conditions in stresses, strains and state parameters as in the experiment should be prescribed for the prediction to achieve any meaning with the comparison. Consequently, $d_{\min }$ for the first experimental point could be set to zero. An economical way to compute $d_{\min }$ is to search for the closest Euclidean distance in Equation (4) in one direction, according to Figure 1. The search proceeds until the closest distance, $d_{k, i}<d_{k, i-1}$ and $d_{k, i}<d_{k, i+1}$, is located. Then the distance $d_{\min }$ to an even closer position is searched for on either side of the prediction point $i$ (the linear segments joining predictions $i-1$ to $i$ and $i$ to $i+1$ ), see the example in Figure 2. In the evaluation of $d_{\min }$, two triangles have to be analysed. A closer distance than $d_{k, i}$ might exist in both the triangles, in only one of the triangles or in none of the triangles. The angle $\alpha$ on each side of the prediction point $i$ in Figure 2 determines the side on which it is necessary to search for a closer distance. In a triangle where the angle $\alpha$ is acute, a closer distance than $d_{k, i}$ can be calculated as the height of a triangle but if the angle $\alpha$ is obtuse no closer distance exists. The closest distance found by analysing the triangles is set to $d_{\min }$. Notice that the search for the next minimum distance to the experimental point $k+1$ in Figure 1 starts from the prediction point $i+1$, i.e. only the remaining part of the theoretical curve is used for the subsequent experimental points.

A requirement for this strategy to work is that the theoretical result has a higher resolution than the experimental result. This should not cause problems since the prediction curve can be computed with any required resolution. Further, the strategy is designed for monotonic loading paths mainly (which are predominant in triaxial testing). To use this approach for cyclic loading, it would be necessary to divide both experimental and theoretical results into adequate segments and measure the norm for each of them separately. However, for e.g. softening material behaviour 


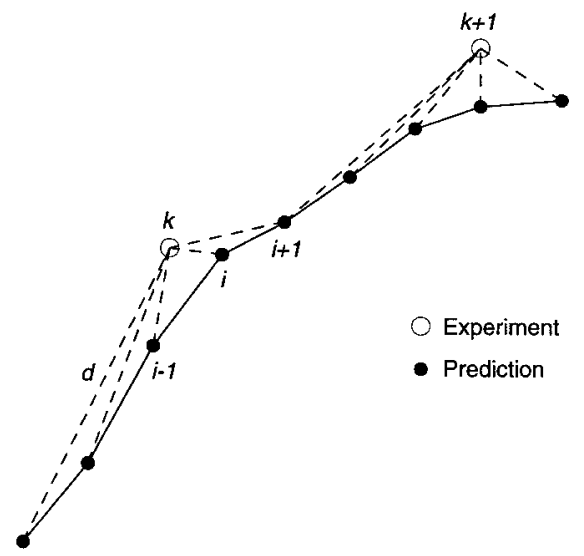

Figure 1. Sketch describing the search algorithm for the closest distances.

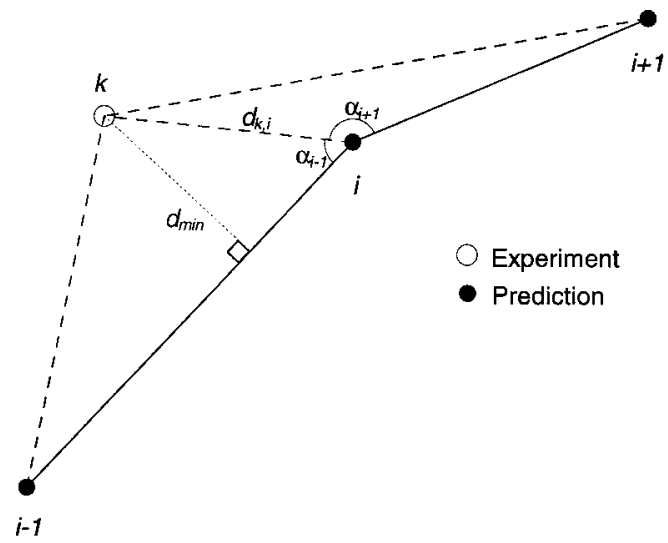

Figure 2. Evaluation of $d_{\min }$.

(just at the start of softening), it has been noticed that the distance $d$ does not always decrease in the beginning of the search for the closest distance. A programmer should pay attention to singular situations like this, by e.g. forcing the distance to decrease before it reaches a minimum.

When the minimum distances for all the experimental points are computed, an absolute individual norm (for a single test) is obtained as

$$
E_{\mathrm{abs}}=w \frac{1}{n+1}\left[\sum_{j=1}^{n} d_{\min }^{j}+d_{t}\right]
$$

where $w$ is a weight factor, $n$ is the number of experimental points (the first not counted), $d_{\min }^{j}$ is the minimum distance for the experimental point $j$ and $d_{t}$ denotes the distance according to Equation (4) between the termination points. The weight factor $w$ is introduced to make it 
possible to characterize the relative importance of all the tests. In most experimental tests, where a constitutive relation is examined, the end state of the test is considered to be specially important (it could e.g. represent a failure state). This is the reason for utilizing the termination distance $d_{t}$ in Equation (7). Obviously, the individual norm formulation leads to higher accuracy when more discrete points are included in the computation.

In addition to the absolute norm (7), another individual norm based on relative deviations between experimental results and the prediction curve is proposed below. The expression in Equation (7) is slightly modified to

$$
E_{\mathrm{rel}}=w \frac{1}{n+1}\left[\sum_{j=1}^{n} \frac{d_{\mathrm{min}}^{j}}{D^{j}}+\frac{d_{t}}{D^{n}}\right]
$$

where $D^{j}$ and $D^{n}$ are determined from the alternatives

$$
\begin{aligned}
& \text { If }\left(\frac{D_{\text {present }}}{D_{\max }} \geqslant \beta\right), \quad D^{j}=D_{\text {present }} \\
& \text { If }\left(\frac{D_{\text {present }}}{D_{\max }}<\beta\right), \quad D^{j}=D_{\max } \cdot \beta
\end{aligned}
$$

where $0 \leqslant \beta \leqslant 1$ is a user-specified constant, $D_{\text {present }}$ is the Euclidean distance for the present experimental point $j$ from an origin

$$
D_{\text {present }}=\left[\frac{1}{s_{0}^{2}} \sum_{i=1}^{3}\left(s_{i}^{\exp }\right)^{2}+\frac{1}{\varepsilon_{0}^{2}} \sum_{i=1}^{3}\left(\varepsilon_{i}^{\text {exp }}\right)^{2}+\frac{1}{u_{0}^{2}}\left(u^{\text {exp }}\right)^{2}\right]^{1 / 2}
$$

and $D_{\max }$ is the maximum Euclidean distance found from an origin for all experimental points included in the computation

$$
D_{\max }=\max \left(D_{\text {present }}\right)
$$

A well-known drawback with a relative norm is that small absolute deviations at low magnitude points can produce extremely large contributions to the computed norm. An extreme case arises if the denominator $D$ in Equation (8) approaches zero which means that the relative norm $E_{\text {rel }}$ approaches infinity. To avoid this drawback, the alternatives in Equation (9) are introduced together with the constant $\beta$, in which a non-zero value of $\beta$ controls the smallest possible magnitude on the denominator $D$.

Both the absolute norm and the relative norm have merits what concerns the interpretation of errors. The selection of norm must be based on what is to be attained with the analysis in question. Therefore, the computer code in the optimization program is designed with these two alternative norms.

The next step is to formulate a final norm, an objective function, based on the individual norms computed for each experimental test included in the optimization. In e.g. a finite element simulation, it is desired that the constitutive model should describe the material behaviour with the highest possible accuracy within the entire stress/strain region of interest, here represented by the experimental tests included in the optimization. From this point of view, it seems to be 
favourable to let the test that currently has the highest individual norm (the worst agreement in prediction) determine the optimization schedule. Mathematically, such a maximum norm is formulated as

$$
F_{\max }=\max _{1 \leqslant l \leqslant m} E^{l}
$$

where $m$ is the number of experimental tests involved in the optimization and $E^{l}$ the norm value for test no. $l$ computed from Equations (7) or (8). However, a deficiency in the formulation of the maximum norm exists. Sometimes the predicted result of the test that provides the maximum norm value, and thereby guides the search, is not sensitive with respect to some of the parameters included in the constitutive model. In such a case, the insensitive parameters do not affect the maximum norm value and could, therefore, attain almost any values inside the bound constraints both during the search and when the search is finished. This information could be misleading and, further, the use of the maximum norm is not particularly appropriate if these insensitive parameters affect other individual norms included in the optimization. Therefore, a combined norm is formulated

$$
F_{\mathrm{comb}}=m \cdot F_{\max }+\sum_{l=1}^{m} E^{l}
$$

with the same notations as in Equation (12). In the above norm, the worst test is still the most important one, but this norm also includes the other individual norms and, when the maximum norm does not change, this one is still able to show improvements. Anyway, because of the different characteristics between the maximum norm and the combined norm, both norms are included in the developed optimization program as an option for the objective function.

\section{THE SEARCH STRATEGY}

In the optimization program developed, it is possible to choose between two different search strategies, one based on Rosenbrock's method [6] and the other on the Simplex method by Nelder and Mead [7]. Both these methods belong to the category of direct search methods in which the search strategy is based only on values of the objective function itself. Alternatively, so-called gradient methods could have been used in which also derivatives of the objective function are utilized. Although gradient methods are commonly regarded as more efficient, the objective function, which is complex and which behaviour is generally unknown, might not always be differentiable and possess continuous derivatives. Hence, to avoid problems and achieve generality, only direct search methods have been considered here.

The Rosenbrock method was chosen since one of the authors have had good experiences of that, Klisinski [5]. In addition, the simplex method was applied because it has manifested itself as one of the more powerful direct search methods presented in the literature, see e.g. References $[1,7,8]$. The efficiency of different search strategies is depending on the type of optimization problem, the number of optimization variables and their starting values, etc., see e.g. Reference [8]. Different search strategies can also approach different local minima for one and the same problem. Hence, two different search methods have been included in the optimization program giving the user an option to switch to the alternative method if the first turns out to be ineffective for some reason during the search. 
In both the Rosenbrock method and the simplex method, an initial set of model parameters and their bound constraints have to be prescribed before the actual optimization can start. It is possible to lock an optional number of these parameters, i.e. the initially prescribed values of the locked parameters are used in the model during the whole optimization. The rest of the model parameters then serve as optimization variables. There are different applications for the locking of parameters in practical optimization. The problem can e.g. be divided into parts, where, for instance, the elastic parameters are determined on the basis of experiments carried out in the elastic region. Thereafter, these elastic parameters are locked and the rest of the model parameters are determined from experiments progressing into the plastic region. With such a strategy (or a similar) a lot of computation time can be saved.

Further, in both methods, it has turned out to be advantageous to normalize all the optimization variables $x^{j}$, for $j=1, \ldots, n$, to the interval $(0,1)$ during the search. This is performed with a linear transformation

$$
\bar{x}^{j}=\frac{x^{j}-x_{l}^{j}}{x_{u}^{j}-x_{l}^{j}}
$$

where $\bar{x}^{j}$ stands for the normalized optimization variable number $j$ and $x_{l}^{j}$ and $x_{\mathbf{u}}^{j}$ are its lower and upper bounds according to Equation (2). Inversely, the transformation back to the optimization variables is given by

$$
x^{j}=\bar{x}^{j}\left(x_{\mathbf{u}}^{j}-x_{l}^{j}\right)+x_{l}^{j}
$$

\subsection{Rosenbrock's method}

In the Rosenbrock method, a set of $n$ mutually orthonormal search direction vectors $\xi_{1}, \xi_{2} \ldots, \xi_{n}$ are defined, together with $n$ associated step lengths $\delta_{1}, \delta_{2} \ldots, \delta_{n}$. Each of the vectors has the dimension $n \times 1$. The number of search direction vectors and their dimension are determined by the number of optimization variables (the number of non-locked model parameters in this case). In the optimization code, the co-ordinate directions are used as an initial choice of search direction vectors, i.e.

$$
\left[\begin{array}{lllll}
\xi_{1} & \xi_{2} & \xi_{3} & \cdots & \xi_{n}
\end{array}\right]=\left[\begin{array}{ccccc}
1 & 0 & 0 & \cdots & 0 \\
0 & 1 & 0 & \cdots & 0 \\
0 & 0 & 1 & \cdots & 0 \\
\vdots & \vdots & \vdots & \ddots & \vdots \\
0 & 0 & 0 & \cdots & 1
\end{array}\right]
$$

Initially, all the step lengths are given the value of a user-specified initial step value $e$, where $0<e<1$. Notice that the introduction of boundaries on the optimization variables and the normalization in Equation (14) makes it possible to prescribe only one initial step value common for all step lengths.

Starting from the initial set of model parameters with an initial step value, the search in the optimization program proceeds according to the following scheme:

1. Compute the objective function $F(\mathbf{x})$, in Equation (12) or (13), for the initial set of model parameters and assign $F_{\min }:=F(\mathbf{x})$, where $:=$ means 'substitute the left side by the right'.

2. Compute $\overline{\mathbf{x}}$ according to Equation (14). 
3. For each $i$ from 1 to $n$ repeat steps 3.1, 3.2 and 3.3.

3.1. Take a step in direction $i, \overline{\mathbf{x}}:=\overline{\mathbf{x}}+\delta_{i} \xi_{i}$

3.1.1. If $\bar{x}^{j}<0$ or $\bar{x}^{j}>1$ for any $j=1, \ldots, n$ then the bound constraints in Equation (2) are violated and the search in this direction is considered as a failure, then

(a) restore $\overline{\mathbf{x}}:=\overline{\mathbf{x}}-\delta_{i} \xi_{i}$

(b) contract and reverse the step length $\delta_{i}:=-\beta \delta_{i}$ where $0<\beta<1$

(c) go to the next search direction in the loop if $i<n$ (step 3.1), else go to the end of the loop (step 4)

3.2. Transform $\overline{\mathbf{x}}$ back to the optimization variables according to Equation (15), and save the result in a backup vector $\mathbf{y}$.

3.3. Compute $F(\mathbf{y})$

3.3.1. If $F(\mathbf{y}) \leqslant F_{\text {min }}$ then the search in this direction is successful and the new point is accepted

(a) $\mathbf{x}:=\mathbf{y}$

(b) $F_{\min }:=F(\mathbf{y})$

(c) the successful step is saved in an associated vector component $d_{i}:=d_{i}+\delta_{i}$

(d) the step length is expanded to $\delta_{i}:=\alpha \delta_{i}$ where $\alpha>1$

3.3.2. Else, if $F(\mathbf{y})>F_{\text {min }}$ the search in this direction is a failure and

(a) $\overline{\mathbf{x}}:=\overline{\mathbf{x}}-\delta_{i} \xi_{i}$ is restored

(b) the step length is contracted and reversed to $\delta_{i}:=-\beta \delta_{i}$ where $0<\beta<1$

4. If a success followed by a failure has been recorded at some time during the iteration for every direction, then

(a) define a new set of orthonormal search direction vectors and reset the vector $\mathbf{d}$

(b) sort the step length vector $\boldsymbol{\delta}$ according to absolute size of its present components, Equations (19)-(21)

(c) continue from step 3.

Else, continue from step 3 .

The basic strategy in Rosenbrock's method is to gradually search for improvement of the objective function in all search directions, and, when the search is successful, an increase in the step length in the particular direction is produced. If the search is unsuccessful, the algorithm will search in the opposite direction using smaller step length during the following loop. This procedure is repeated until the search is considered to be ineffective, when a success followed by a failure has been recorded at some time during the iteration for every direction (the same criterion as Rosenbrock [6] originally suggested). Then, a new set of search directions is defined and the search starts again with these new directions and new step lengths.

To obtain new search directions $\xi^{1}$, step 4(a) in the scheme above, a matrix $\mathbf{A}$ is established

$$
\mathbf{A}=\left[\begin{array}{lllll}
\mathbf{A}_{1} & \mathbf{A}_{2} & \mathbf{A}_{3} & \cdots & \mathbf{A}_{n}
\end{array}\right]
$$

where the subvectors

$$
\begin{aligned}
& \mathbf{A}_{1}=\mathrm{d}_{1} \xi_{1}^{0}+\mathrm{d}_{2} \xi_{2}^{0}+\mathrm{d}_{3} \xi_{3}^{0}+\cdots+\mathrm{d}_{n} \xi_{n}^{0} \\
& \mathbf{A}_{2}=\quad \mathrm{d}_{2} \xi_{2}^{0}+\mathrm{d}_{3} \xi_{3}^{0}+\cdots+\mathrm{d}_{n} \xi_{n}^{0} \\
& \mathbf{A}_{3}=\quad \mathrm{d}_{3} \xi_{3}^{0}+\cdots+\mathrm{d}_{n} \xi_{n}^{0} \\
& \text {...... } \\
& \mathbf{A}_{n}=\quad d_{n} \xi_{n}^{0}
\end{aligned}
$$


are defined from the summation of all the successful steps $d_{i}$ in the direction $\xi_{i}^{0}$ (step 3.3.1(c) above) and the present search directions $\xi^{0}$. Then the modified Gram-Schmidt algorithm (see e.g. Reference [9]) is used to generate a new orthonormal sequence $\left[\begin{array}{lllll}\xi_{1}^{1} & \xi_{2}^{1} & \xi_{3}^{1} & \ldots & \xi_{n}^{1}\end{array}\right]$ of search direction vectors out from the linearly independent sequence $\left[\begin{array}{lllll}\mathbf{A}_{1} & \mathbf{A}_{2} & \mathbf{A}_{3} & \cdots & \mathbf{A}_{n}\end{array}\right]$. This orthonormalization results in a sequence of vectors where $\xi_{1}^{1}$ lies along the direction of fastest advance, $\xi_{2}^{1}$ along the best direction which can be found normal to $\xi_{1}^{1}$, and so on.

The reason for utilizing the modified Gram-Schmidt algorithm instead for the classical Gram-Schmidt process, suggested by Rosenbrock [6], is that the former has shown to possess superior numerical properties, see Reference [10]. Further, based on the conclusions in the paper by Rice, a reinforcement of the vectors $\xi^{1}$ is used in the optimization program, i.e. one repeated application of the modified Gram-Schmidt algorithm.

A problem, not discussed by Rosenbrock [6], is how to associate the step lengths $\delta$ with the new search directions. The absolute magnitude of the present step lengths in the computation stage, after the definition of new search directions, reflects the progress of optimization, but the association between $\delta_{i}$ and $\xi_{i}$ no longer exists. In the program (step 4(b) above) this problem has been tackled by taking the absolute value of all the elements in the present step length vector $\boldsymbol{\delta}^{0}$

$$
\delta_{i}^{0}:=\left|\delta_{i}^{0}\right|, \quad i=1, \ldots, n
$$

and sorting them in decreasing order into a new step length vector

$$
\boldsymbol{\delta}^{1}=\left[\begin{array}{lllll}
\delta_{1}^{1} & \delta_{2}^{1} & \delta_{3}^{1} & \cdots & \delta_{n}^{1}
\end{array}\right]
$$

where thus

$$
\delta_{1}^{1} \geqslant \delta_{2}^{1} \geqslant \delta_{3}^{1} \geqslant \cdots \geqslant \delta_{n}^{1}>0
$$

Hereby, $\delta_{1}^{1}$, which has the largest value, will be associated with the direction of 'fastest advance' $\xi_{1}^{1}$, and $\delta_{2}^{1}$, with the second highest value, will be associated with $\xi_{2}^{1}$ the second most promising direction, and so on. It was observed during the development of the optimization program that this sorting strategy considerably improves the efficiency of the algorithm compared to the alternative of just keeping the step lengths $\boldsymbol{\delta}^{0}$ from the previous loop.

Rosenbrock [6] performed a set of computations, on a mathematical function of two variables, in order to determine the best combination of values of the step adjustment parameters $\alpha$ (in step 3.3.1(d)) and $\beta$ (in steps 3.1.1(b) and 3.3.2(b)) and arrived at the values $\alpha=3$ and $\beta=0.5$. However, it could be expected that the optimal combination of $\alpha$ and $\beta$ is highly dependent on the type of problem to which the algorithm is applied. Therefore, the values of $\alpha$ and $\beta$ utilized in the current optimization routine were evaluated from an optimization problem representative for the type of optimization discussed in this paper, see Appendix A. From this study, it was concluded that the optimal combination for an efficient algorithm was $\alpha=1.9$ and $\beta=0.8$.

The initial step value, $0<e<1$, is a parameter that affects the efficiency of the algorithm, which is shown in Appendix A. Because the best choice of $e$ could be expected to greatly depend on the optimization problem under inspection, even for the type of optimization discussed here, it was considered to be favourable to treat $e$ as a user-specified input parameter. It is also possible, for different initial step values, to direct the search towards different local minima (if such do exist). This is another, reason to let $e$ be an input parameter. However, from the experience with the algorithm, it seems to be favourable to choose the initial step value from the lower portions of 
the interval $0<e<1$ as a first attempt, but not 'to close' to the lower boundary. For the optimization with the optimal combination $\alpha=1.9$ and $\beta=0.8$, in Appendix A, it seems that the best results are obtained for $0.2 \leqslant e \leqslant 0.45$.

In order to terminate Rosenbrock's search algorithm, three different conditions are proposed. These are the maximum number of objective function computations (the first computation in step 1 not counted), the maximum number of unsuccessful objective function computations and a criterion for sufficient optimization accuracy. The first condition limits the execution time for the search since it mainly depends on the number of objective function computations. The second condition terminates the search when it seems to be ineffective, e.g. when a minimum or a point close to it has been reached. In the third condition, the search is terminated when the absolute value of all the step lengths $\left|\delta_{i}\right|$ for $i=1, \ldots, n$ is less than the prescribed accuracy after one finished loop (step 3) in the algorithm. All these termination conditions are prescribed before the search starts and the one that is first reached terminates the search.

\subsection{The simplex method}

In the simplex method, the search for the minimum of the objective function proceeds by comparing the magnitude of objective function values at the vertices of a geometric figure (named a simplex) in an Euclidean space spanned by the normalized optimization variables (Equation (14)). For a problem with $n$ optimization variables, the simplex is composed by $n+1$ points. The simplex geometrically corresponds to a segment for $n=1$, a triangle for $n=2$ and a tetrahedron for $n=3$. By successively exchanging the point with the highest objective function value for a new improved point, the simplex will move in the space in the direction of a minimum. In the version by Nelder and Mead [7], utilized in the optimization program, the simplex also rescales itself during the search according to the local geometry of the objective function. This is accomplished by the three operations named reflection, expansion and contraction, as illustrated in Figure 3 for a problem with two variables. These operations are mathematically defined in the algorithm presented below.

During the development of the optimization routine, it turned out that the efficiency of the simplex method was highly influenced by the design of the starting simplex. A new strategy has been worked out based on the regular simplex of unit edge proposed by Spendley et al. [11]. It was believed to be favourable to let the user prescribe the size of the starting simplex and thus, to some extent, make it possible to reach different local minima (if such exist) from the same initial
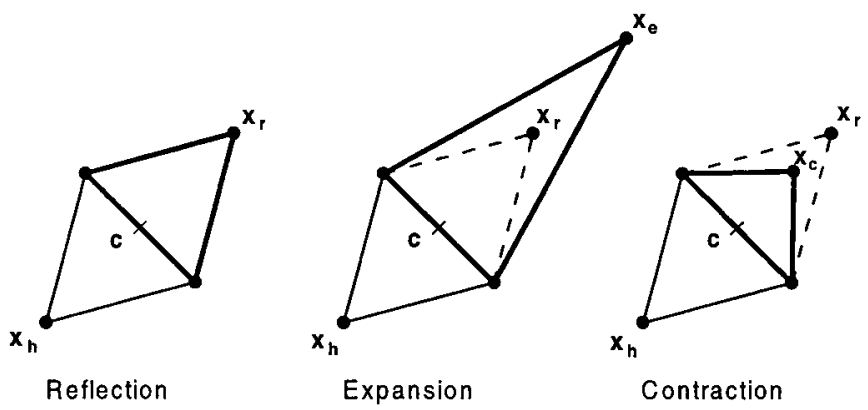

Figure 3. Reflection, expansion and contraction moves on an $n=2$ simplex. 


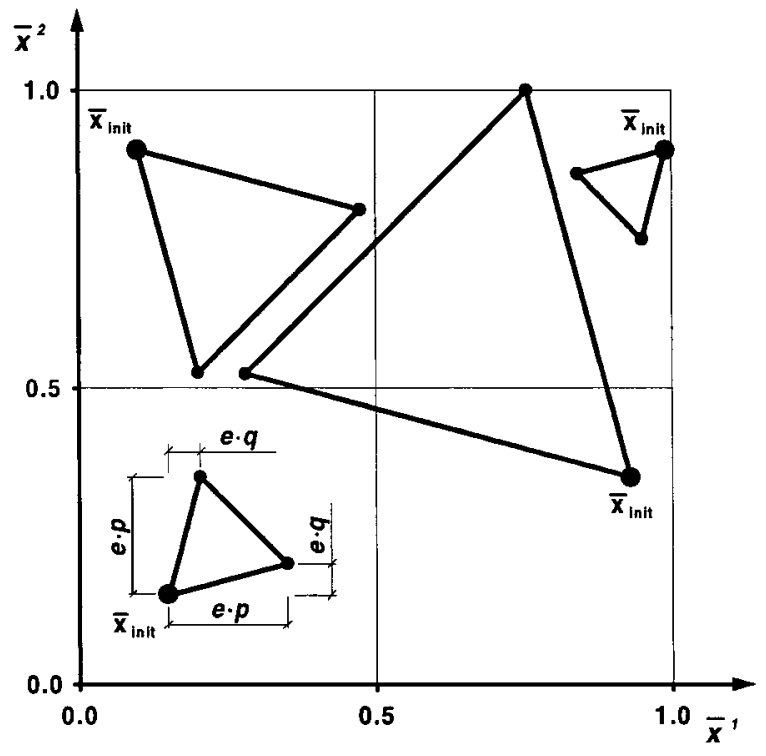

Figure 4. The starting simplex strategy, exemplified on an $n=2$ simplex.

set of model parameters. To facilitate the prescription of a large starting simplex, the orientation of the simplex has been made a function of the location in the space of the initial set of normalized optimization variables $\overline{\mathbf{x}}_{\text {init }}$. The strategy is shown in Figure 4 for an $n=2$ simplex.

The initial guess $\overline{\mathbf{x}}_{\text {init }}$ is always included as a point in the starting simplex. The additional points in the simplex, relative to the initial guess $\overline{\mathbf{x}}_{\text {init }}$, are mathematically constructed by premultiplying the $n \times n$ design matrix

$$
\mathbf{D}_{m}=\left[\begin{array}{ccccc}
p & q & q & \cdots & q \\
q & p & q & \cdots & q \\
q & q & p & \cdots & q \\
\vdots & \vdots & \vdots & \ddots & \vdots \\
q & q & q & \cdots & p
\end{array}\right]
$$

where

$$
p=\frac{1}{n \sqrt{2}}[(n-1)+\sqrt{n+1}]
$$

and

$$
q=\frac{1}{n \sqrt{2}}[\sqrt{n+1}-1]
$$


with a user-defined scalar $0<e<1$, expressing the Euclidean distances between the vertices of the simplex and an $n \times n$ diagonal matrix

$$
\mathbf{I}_{d}=\left[\begin{array}{ccccc}
a_{11} & 0 & 0 & \cdots & 0 \\
0 & a_{22} & 0 & \cdots & 0 \\
0 & 0 & a_{33} & \cdots & 0 \\
\vdots & \vdots & \vdots & \ddots & \vdots \\
0 & 0 & 0 & \cdots & a_{n n}
\end{array}\right]
$$

that defines the orientation of the simplex by the identification process

$$
\begin{aligned}
& \text { for } j=1, \ldots, n, \text { do } \\
& \text { if } 0<\bar{x}_{\text {init }}^{j} \leqslant 0.5 \text { then } \\
& \quad a_{j j}:=1 \\
& \text { else if } 0.5<\bar{x}_{\text {init }}^{j}<1 \text { then } \\
& a_{j j}:=-1
\end{aligned}
$$

end if

end

where $\bar{x}_{\text {init }}^{j}$ for $j=1, \ldots, n$ are the components of $\overline{\mathbf{x}}_{\text {init }}$. The columns of the resulting matrix $\mathbf{D}_{\text {start }}=e \mathbf{I}_{d} \mathbf{D}_{m}$ give then the contributions relative to the initial guess $\overline{\mathbf{x}}_{\text {init }}$ and thus defines the starting simplex. However, if any of the points ends up outside the space defined by the normalized optimization variables, the program uses the largest possible regular simplex as the starting simplex (as indicated in Figure 4).

With the starting simplex defined, the search in the optimization program proceeds according to the following scheme:

1. Let $\overline{\mathbf{x}}_{i}$ for $i=0, \ldots, n$ define the vertices of the starting simplex, represented as points in the normalized space. Compute the objective function value associated with each vertex $F_{i}\left(\mathbf{x}_{i}\left(\overline{\mathbf{x}}_{i}\right)\right)$ by first transforming each component in the vector $\overline{\mathbf{x}}_{i}$ back to their physical counterpart $\mathbf{x}_{i}$, according to Equation (15).

2. Carry out the search by repeating steps $2.1-2.5$ until any termination condition is reached.

2.1. From the objective function values at each vertex of the current simplex, $F_{i}$ for $i=0, \ldots, n$, determine the suffixes $h$ and $l$ that are associated with the maximum function value $F_{h}=\max _{i=0 \text { to } n}\left(F_{i}\right)$ and the minimum function value $F_{l}=\min _{i=0 \text { to } n}\left(F_{i}\right)$, respectively.

2.2. Compute the centroid $\mathbf{c}$ of all the points $\overline{\mathbf{x}}_{i}$ except the point corresponding to the suffix $h$, as $\mathbf{c}=(1 / n) \sum_{\substack{i=0 \\ i \neq h}}^{n} \overline{\mathbf{x}}_{i}$.

2.3. Make a reflection move $\overline{\mathbf{x}}_{\mathrm{r}}=\mathbf{c}+\gamma\left(\mathbf{c}-\overline{\mathbf{x}}_{\mathrm{h}}\right)$, where $\gamma>0$ is the reflection coefficient. If any component in the vector $\overline{\mathbf{x}}_{\mathrm{r}}$ ends up outside the bound constraints, substitute that component with the value of the exceeded bound, i.e. if $\bar{x}_{\mathrm{r}}^{j}<0$ set $\bar{x}_{\mathrm{r}}^{j}=0$ or if $\bar{x}_{\mathrm{r}}^{j}>1$ set $\bar{x}_{\mathrm{r}}^{j}=1$.

2.4. Compute the objective function value for the reflected point, $F_{\mathrm{r}}\left(\mathbf{x}_{\mathrm{r}}\left(\overline{\mathbf{x}}_{\mathrm{r}}\right)\right)$. 
2.5. Select the proper case out from the three possible cases, described in steps 2.5.1-2.5.3. 2.5.1. If $F_{\mathrm{r}}<F_{l}$ then $\overline{\mathbf{x}}_{\mathrm{r}}$ is a new best point and the direction of the reflection is examined further by an expansion move $\overline{\mathbf{x}}_{\mathrm{e}}=\mathbf{c}+\zeta\left(\overline{\mathbf{x}}_{\mathrm{r}}-\mathbf{c}\right)$, where $\zeta>1$ is the expansion coefficient. Followed by a check of the bound constraints, if $\bar{x}_{\mathrm{e}}^{j}<0$ set $\bar{x}_{\mathrm{e}}^{j}=0$ or if $\bar{x}_{\mathrm{e}}^{j}>1$ set $\bar{x}_{\mathrm{e}}^{j}=1$, and a computation of the associated objective function value $F_{\mathrm{e}}\left(\mathbf{x}_{\mathrm{e}}\left(\overline{\mathbf{x}}_{\mathrm{e}}\right)\right)$.

(a) If $F_{\mathrm{e}}<F_{\mathrm{r}}$, the expansion is successful and the exchange $\overline{\mathbf{x}}_{h}:=\overline{\mathbf{x}}_{\mathrm{e}}$ and $F_{\mathrm{h}}:=F_{\mathrm{e}}$ is performed.

(b) Otherwise, if $F_{\mathrm{e}} \geqslant F_{\mathrm{r}}$, the expansion is unsuccessful and the exchange $\overline{\mathbf{x}}_{h}:=\overline{\mathbf{x}}_{\mathrm{r}}$ and $F_{h}:=F_{\mathrm{r}}$ is performed.

2.5.2. If $F_{\mathrm{r}}<F_{\mathrm{i}}$ for some $i=0, \ldots, n$ except $i=h$, the reflection led to an improved simplex and the exchange $\overline{\mathbf{x}}_{h}:=\overline{\mathbf{x}}_{\mathrm{r}}$ and $F_{h}:=F_{\mathrm{r}}$ is performed.

2.5.3. If $F_{\mathrm{r}}>F_{i}$ for all $i=0, \ldots, n$ except $i=h$, the reflection resulted in a new worst point and a contraction move $\overline{\mathbf{x}}_{c}=\mathbf{c}+\eta\left(\overline{\mathbf{x}}_{h}-\mathbf{c}\right)$ if $F_{h}<F_{\mathrm{r}}$ or $\overline{\mathbf{x}}_{c}=\mathbf{c}+\eta\left(\overline{\mathbf{x}}_{\mathrm{r}}-\mathbf{c}\right)$ if $F_{h} \geqslant F_{\mathrm{r}}$ is performed. Here, the factor $0<\eta<1$ stands for the contraction coefficient. This is followed by a computation of the associated value of the objective function $F_{c}\left(\mathbf{x}_{c}\left(\overline{\mathbf{x}}_{c}\right)\right)$.

(a) If now $F_{c}<\min \left(F_{h}, F_{\mathrm{r}}\right)$, the contraction is considered to be successful and the exchange $\overline{\mathbf{x}}_{h}:=\overline{\mathbf{x}}_{c}$ and $F_{h}:=F_{c}$ is performed.

(b) Else, if $F_{c} \geqslant \min \left(F_{h}, F_{\mathrm{r}}\right)$, a comprehensive contraction is carried out by replacing all the points $\overline{\mathbf{x}}_{i} i=0, \ldots, n$ except the best $\overline{\mathbf{x}}_{i=l}$ by $\left(\overline{\mathbf{x}}_{i}+\overline{\mathbf{x}}_{l}\right) / 2$ and recompute their associated objective function values $F_{i}\left(\mathbf{x}_{i}\left(\overline{\mathbf{x}}_{i}\right)\right)$.

To terminate the search in the simplex method, two different conditions are used: the maximum number of computations of the objective function (also utilized in the Rosenbrock algorithm) and a criterion, adopted by Nelder and Mead [7], that terminates the search when the standard deviation of the objective function values at each vertex of the current simplex falls below a pre-set value $\varepsilon$, that is

$$
\left(\sum_{i=0}^{n} \frac{\left(F_{i}-\bar{F}\right)^{2}}{n}\right)^{1 / 2}<\varepsilon
$$

where

$$
\bar{F}=\frac{1}{n+1} \sum_{i=0}^{n} F_{i}
$$

is the average function value. The point associated with the smallest objective function value, at the time when the search is terminated, is used as the solution of the optimization problem.

Before the simplex method can be applied for practical optimization, the values of the reflection coefficient $\gamma$ (in step 2.3), the expansion coefficient $\zeta$ (in step 2.5.1) and the contraction coefficient $\eta$ (in step 2.5.3) have to be assigned. As a conclusion of their testing, Nelder and Mead [7] suggested the values of $\gamma=1, \zeta=2$ and $\eta=0.5$. Other 'optimal combinations' have been reported in the literature, see e.g. Reference [12] in which the combination $\gamma=2, \zeta=2.5$ and $\eta=0.25$ showed to be somewhat superior for their testing program compared to the combination by Nelder and Mead [7]. To obtain a combination of values representative for the type of optimization discussed here, an evaluation of the coefficients was performed based on the same 
optimization problem as in Appendix A. From this study, presented in Appendix B, it was concluded that the values $\gamma=1, \zeta=2$ and $\eta=0.6$ was optimal and should be used in the optimization program.

It is seen from Appendix B that the size $0<e<1$ of the starting simplex do affect the efficiency of the algorithm. The best choice of $e$ could be expected to greatly depend on the optimization problem under inspection, but it has been observed that a choice of $e$ to close to the lower boundary should generally be avoided, at least as a first attempt. For the optimizations with the optimal combination $\gamma=1, \zeta=2$ and $\eta=0.6$, in Appendix B, almost all values of $e$ greater than 0.12 gave good results.

\section{OPTIMIZATION EXAMPLES}

The practical outcome of an optimization clearly depends on the ability of the chosen model to predict the real soil behaviour. If the chosen model is not able to predict the soil behaviour 'sufficiently accurate', there is no meaning to carry out or continue the optimization. With that in

mind, it is obviously important to have reasonable knowledge about the range of application of the available models.

In this paper, it is the numerical capacity of the optimization routine that is essential to examine. To accomplish this in a proper way, an optimization problem is constructed, Section 5.1, where the uncertainty of the prediction ability of the chosen model is removed from the problem by using constitutive simulations with an in advance known set of model parameters as simulated experimental result. The actual optimization problem is then created by choosing a different set of model parameters as a starting point for the optimization. Thereby, it is no doubt about that the model is able to simulate the 'experimental result' and the proper minimum is known in advance. Optimizations against the simulated experimental result are discussed in Section 5.1, and in Section 5.2 the performance of the optimization routine is examined when the simulated experimental result is subjected to perturbations. In addition, optimization examples against real triaxial tests are shown and discussed in Section 5.3.

\subsection{Optimization against simulated experimental result}

The constitutive model chosen for this example is the generalized cam clay model, described in Reference [13]. The model is based on the classical modified cam clay concept (see e.g. Reference [14]) and generalized with respect to the yield function, the flow rule and the hardening rule. Seven parameters are included in the model, i.e.

$\kappa^{*} \quad$ the slope of the unloading-reloading line in the $\varepsilon_{p}-\ln p^{\prime}$ plane, where $\varepsilon_{p}$ is the volumetric strain and $p^{\prime}$ the effective mean stress

$v^{\prime} \quad$ Poisson's ratio

$\lambda^{*} \quad$ the slope of the isotropic compression line in the $\varepsilon_{p}-\ln p^{\prime}$ plane

$D$ deviatoric hardening parameter

$M \quad$ the slope of the critical state cone

$N$ parameter for a non-associated flow rule

$e_{r} \quad$ parameter for the yield surface dependence on the third deviatoric stress invariant. 
Table I. Loading programs.

\begin{tabular}{|c|c|c|c|c|c|c|c|c|c|c|c|c|}
\hline \multirow[t]{2}{*}{ Test } & \multirow{2}{*}{$\begin{array}{c}\text { Drained } \\
{[0] \text { or }} \\
\text { undrained } \\
{[1]}\end{array}$} & \multicolumn{5}{|c|}{ Initial conditions } & \multicolumn{6}{|c|}{ End conditions of control parameters } \\
\hline & & $\begin{array}{c}p_{c}^{\prime} \\
(\mathrm{kPa})\end{array}$ & $\begin{array}{c}S_{1} \\
(\mathrm{kPa})\end{array}$ & $\begin{array}{c}S_{2} \\
(\mathrm{kPa})\end{array}$ & $\begin{array}{c}S_{3} \\
(\mathrm{kPa})\end{array}$ & $\begin{array}{c}u \\
(\mathrm{kPa})\end{array}$ & $\begin{array}{c}s_{1} \\
(\mathrm{kPa})\end{array}$ & $\begin{array}{c}S_{2} \\
(\mathrm{kPa})\end{array}$ & $\begin{array}{c}S_{3} \\
(\mathrm{kPa})\end{array}$ & $\begin{array}{c}\varepsilon_{1} \\
(\%)\end{array}$ & $\begin{array}{c}\varepsilon_{2} \\
(\%)\end{array}$ & $\begin{array}{c}\varepsilon_{3} \\
(\%)\end{array}$ \\
\hline 1 & 0 & 50 & 50 & 50 & 50 & 0 & - & 50 & 50 & 10 & - & - \\
\hline 2 & 1 & 50 & 50 & 50 & 50 & 0 & - & 50 & 50 & 5 & - & - \\
\hline 3 & 0 & 75 & 50 & 50 & 50 & 0 & - & 50 & 50 & -10 & - & - \\
\hline 4 & 1 & 75 & 50 & 50 & 50 & 0 & - & 50 & 50 & -5 & - & - \\
\hline 5 & 0 & 250 & 50 & 50 & 50 & 0 & - & 50 & 50 & 10 & - & - \\
\hline 6 & 1 & 250 & 50 & 50 & 50 & 0 & - & 50 & 50 & 5 & - & - \\
\hline 7 & 0 & 50 & 50 & 50 & 50 & 0 & - & - & - & 8 & -4 & 4 \\
\hline 8 & 0 & 50 & 50 & 50 & 50 & 0 & 130 & 90 & 75 & - & - & - \\
\hline 9 & 1 & 50 & 50 & 50 & 50 & 0 & 75 & 25 & - & - & - & 0 \\
\hline 10 & 1 & 50 & 50 & 50 & 50 & 0 & 110 & 80 & 70 & - & - & - \\
\hline
\end{tabular}

To obtain a real challenge for the numerical process in the optimization routine developed, a large problem with ten different types of tests is constructed as a basis for the optimization, Table I. The isotropic effective mean stress $p_{c}^{\prime}$ (in Table I) is the hardening parameter which represents the reference size of the yield surface. Tests 1-6 describe conventional triaxial tests while tests $7-10$ describe experiments that can only be performed in a true triaxial apparatus. In the tests, a linear variation of the control parameters between the initial and final values is assumed.

In the example, the 'experimental result' is obtained by simulating the tests in Table I with the parameters $\kappa^{*}=0.02, v^{\prime}=0.3, \lambda^{*}=0.2, D=1.5, M=1.2, N=0.8$ and $e_{r}=0.65$. These parameters will then give the in advance known solution of the problem (the global minimum). The parameter values are mainly chosen from Alawaji et al. [13]. Further, the problem is designed in such a way that the elastic parameters $\kappa^{*}=0.02$ and $v^{\prime}=0.3$ are locked, whereas the rest of the parameters $\lambda^{*}, D, M, N$ and $e_{r}$ serve as optimization variables. As a starting point for the optimization, an initial set of optimization variables $\lambda^{*}=0.1, D=0.5, M=1.0, N=1.0$ and $e_{r}=0.9$ is chosen. Reasonable lower and upper bounds are chosen as $0.05 \leqslant \lambda^{*} \leqslant 0.4$, $0.1 \leqslant D \leqslant 2.0,0.8 \leqslant M \leqslant 1.4,0.1 \leqslant N \leqslant 1.4$ and $0.5 \leqslant e_{r} \leqslant 1.0$. Notice, that the loading programs in Table I are constructed in such a way that each optimization variable will have an influence on the result from at least one prediction. The integration of the tests in Table I are performed with the explicit Forward Euler integration algorithm containing a method to correct for the yield surface drift [4]. In both the computation of the 'experimental result' and in theoretical predictions during the optimization, the integration is carried out with 200 integration steps for each test. Since the computation of the individual norm requires higher resolution for the theoretical result than that of the experimental result, every fifth point obtained in the computation of the 'experimental result' is saved for individual norm computations. This means that altogether a resolution of 40 discrete points for the 'experimental result' and 200 discrete points for the theoretical result are involved in computations of the individual norm.

The optimization problem chosen has been used for the evaluation of the step adjustment parameters in Rosenbrock's method. Appendix A, as well as of the reflection, expansion and contraction coefficients in the Simplex method, Appendix B. In these optimizations, the combined 
Table II. Comparison of search strategies.

\begin{tabular}{lcccc}
\hline Search strategy & \multicolumn{4}{c}{ Statistical data for $F_{\min }^{200}(\%)$} \\
\cline { 2 - 5 } & Average value & Std. deviation & Min. value & Max. value \\
\hline Rosenbrock's method & 39.6 & 30.2 & 1.34 & 96.6 \\
The simplex method & 3.7 & 8.9 & 0.031 & 88.2 \\
\hline
\end{tabular}

norm (equation (13)) has been utilized as the objective function with the absolute norm (equation (7)) without weighting $\left(w_{i}=1.0\right.$ for test $\left.i=1, \ldots, 10\right)$ of the individual tests. The statistical data from the cases in Table A1 and in Table B3, respectively, representing optimizations with the parameter values that were finally chosen, are shown in Table II for comparison. The symbol $F_{\text {min }}^{200}$ stands for the smallest value of the objective function, obtained after 200 computations of the objective function in the search algorithms. As a basis for the statistical data, 100 optimizations for Rosenbrock's method and 400 optimizations for the simplex method were performed. The initial step length for Rosenbrock's method and the size of the initial simplex for the simplex method, both denoted as $e$, were randomly generated between $0<e<1$ for each new optimization. In all other aspects, the optimizations, in Table II, were performed under the same conditions for each search strategy, respectively.

The statistical data in Table II show that the choice of $e$-value, for both the search strategies, influences the optimization result. This was, however, the main reason for treating $e$ as a userspecified input parameter. To get an idea of the average improvements that could be expected with a randomly chosen $e$-value, after 200 computations of the objective function, the average value of $F_{\min }^{200}$ can be compared to the objective function value for the initial set of model parameters, that is 341.5 per cent. It can thus be concluded that it is reasonable to expect considerable improvements, independent of the chosen $e$-value, for both the search strategies in this example. However, a faster convergence in average towards the minimum was obtained for the simplex method compared to the Rosenbrock method.

So far, only values of the objective function have been studied. This is a scalar which sometimes can be difficult to interpret if its value at the same time is not put in relation to the current set of optimization variables, to the individual norms and to actual stress-strain plots. In the following, a closer look will be taken on the optimizations in Table II, leading to the minimum values of $F_{\min }^{200}$. This study is presented in Tables III and IV and Figure 5 for Rosenbrock's method and in Tables V and VI and Figure 6 for the simplex method. In addition, for the optimizations that led to maximum values of $F_{\min }^{200}$, a discussion will be carried out around the circumstances that caused those higher values.

In Tables III and V, the changes of the optimization variables during the course of optimization can be followed. These results were obtained with the initial step length $e=0.680$ for Rosenbrock's method and the size of the initial simplex $e=0.303$ for the simplex method. For Rosenbrock's method, Table III, gradual improvements are obtained during the whole course of optimization and after 1000 computations of the objective function, the global minimum is reached almost to the last decimal presented for the optimization variables. For the simplex method, Table $\mathrm{V}$, a local minimum is reached after 400 computations of the objective function. This local minimum is, however, located so close to the global minimum that the error has no 
Table III. The Rosenbrock method-optimization variables.

\begin{tabular}{|c|c|c|c|c|c|c|}
\hline \multirow{2}{*}{$\begin{array}{l}\text { Number of } \\
\text { computations } \\
\text { of } F_{\text {comb }}\end{array}$} & \multirow{2}{*}{$\begin{array}{l}F_{\min } \\
(\%)\end{array}$} & \multicolumn{5}{|c|}{ Optimization variables } \\
\hline & & $\lambda^{*}$ & $D$ & $M$ & $N$ & $e_{r}$ \\
\hline 0 (initial) & 341.519 & 0.1 & 0.5 & 1.0 & 1.0 & 0.9 \\
\hline 25 & 91.6668 & 0.252332 & 1.97051 & 1.10696 & 0.460391 & 0.663989 \\
\hline 50 & 69.2279 & 0.206637 & 1.52940 & 1.15429 & 0.782443 & 0.643529 \\
\hline 75 & 18.6460 & 0.206637 & 1.54665 & 1.21397 & 0.834621 & 0.652103 \\
\hline 100 & 9.20714 & 0.202932 & 1.55376 & 1.18986 & 0.815918 & 0.649030 \\
\hline 150 & 3.74938 & 0.202932 & 1.53056 & 1.19962 & 0.799397 & 0.649203 \\
\hline 200 & 1.34061 & 0.201023 & 1.51477 & 1.19938 & 0.801974 & 0.649998 \\
\hline 300 & 0.0881827 & 0.200018 & 1.50008 & 1.19992 & 0.800329 & 0.650025 \\
\hline 400 & 0.0332130 & 0.200023 & 1.50028 & 1.20001 & 0.800326 & 0.649992 \\
\hline 500 & 0.0126827 & 0.199992 & 1.49990 & 1.20001 & 0.800193 & 0.649998 \\
\hline 1000 & 0.000355206 & 0.200000 & 1.50000 & 1.20000 & 0.800005 & 0.650000 \\
\hline
\end{tabular}

practical meaning. Before this local minimum was reached, the simplex method turned out to be more efficient than the Rosenbrock method. The fact that the global minimum is found with such a good precision with Rosenbrock's method (and almost with the simplex method) proofs that the mathematical strategy for the computation of the individual norm works remarkably good. It should be noticed that the large number of computations of the objective function was performed to get an idea of the possible precision. From the practical point of view, the optimizations could have been stopped, with a sufficient accuracy, much earlier.

To show how much the predictions of the individual tests deviate from their associated 'experimental result' during the course of optimization, values of the absolute individual norm for each test are given in Tables IV and VI. In these tables, the test that currently has the largest individual norm value $F_{\max }$, in Equation (13), and thus is the most important for the optimization schedule, has been printed in italics. It is seen, in both tables, that different tests represent $F_{\text {max }}$ during the process of optimization. Further, a comparison between Tables IV and VI displays a different $F_{\max }$ representation between the search strategies. This is an evidence for the fact that the search strategies work differently, which means that they not necessarily need to approach the same local minimum (if such exist). This is a fact that clearly motivates the use of more than one search strategy in an optimization program.

In Figures 5 and 6, progresses during the course of optimization are visualized, for one test for each strategy, in plots with the invariants

$$
\begin{aligned}
p^{\prime} & =\left(\sigma_{1}+\sigma_{2}+\sigma_{3}\right) / 3 \\
q & =\left(\frac{\left(\sigma_{1}-\sigma_{2}\right)^{2}+\left(\sigma_{2}-\sigma_{3}\right)^{2}+\left(\sigma_{1}-\sigma_{3}\right)^{2}}{2}\right)^{1 / 2} \\
\varepsilon_{p} & =\varepsilon_{1}+\varepsilon_{2}+\varepsilon_{3} \\
\varepsilon_{q} & =\frac{2^{1 / 2}}{3}\left(\left(\varepsilon_{1}-\varepsilon_{2}\right)^{2}+\left(\varepsilon_{2}-\varepsilon_{3}\right)^{2}+\left(\varepsilon_{1}-\varepsilon_{3}\right)^{2}\right)^{1 / 2}
\end{aligned}
$$




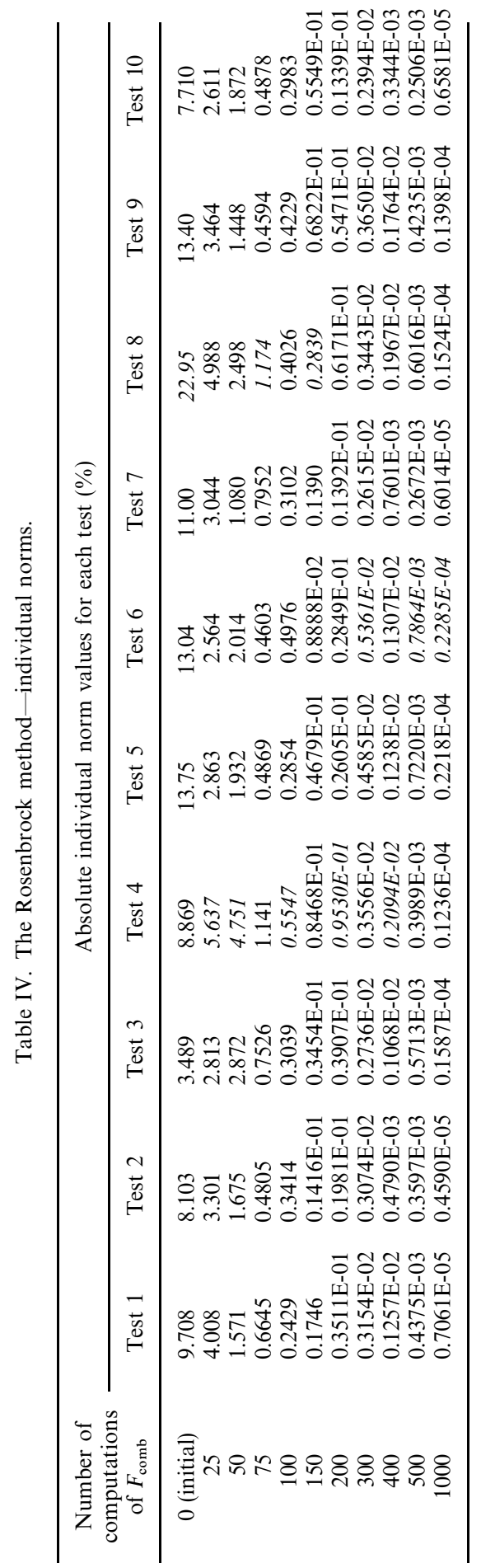



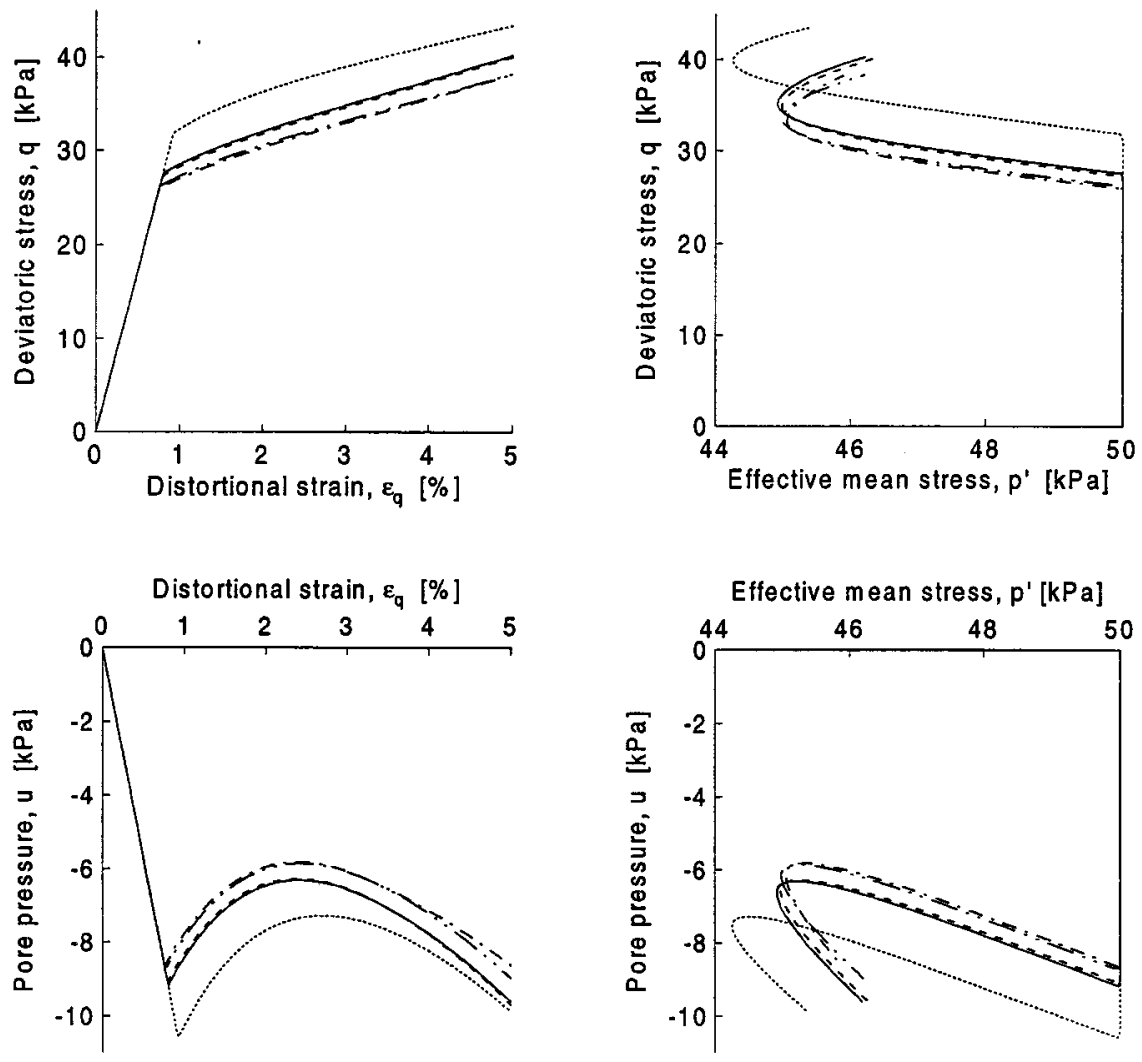

Number of objective function computations

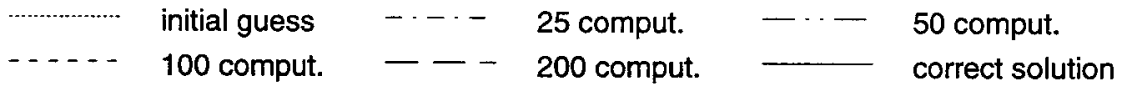

Figure 5. The Rosenbrock method-progresses during optimization for test 4 in Table IV.

where $\sigma_{1}, \sigma_{2}$ and $\sigma_{3}$ are the principal effective stresses and $\varepsilon_{1}, \varepsilon_{2}$ and $\varepsilon_{3}$ are the principal strains. Test no. 4, which obtained the largest individual norm value $F_{\max }$ most times during the optimization with Rosenbrock's method in Table IV, has been chosen for the plots in Figure 5. In a similar way, test no. 8 in the optimization with the simplex method in Table VI, has been chosen for Figure 6. Test no. 4 describes a conventional undrained triaxial extension test on a lightly overconsolidated soil and test no. 8, a drained stress controlled true triaxial test on a normally consolidated soil. With the initial guess as a starting point, it is seen in Figures 5 and 6 that gradual improvements towards the correct solution are obtained during the optimizations. As can be seen in Figure 5, the response seems to coincide with the correct solution after 200 computations of the objective function while, in Figure 6, the response almost coincide with the correct solution already after 100 computations of the objective function. 

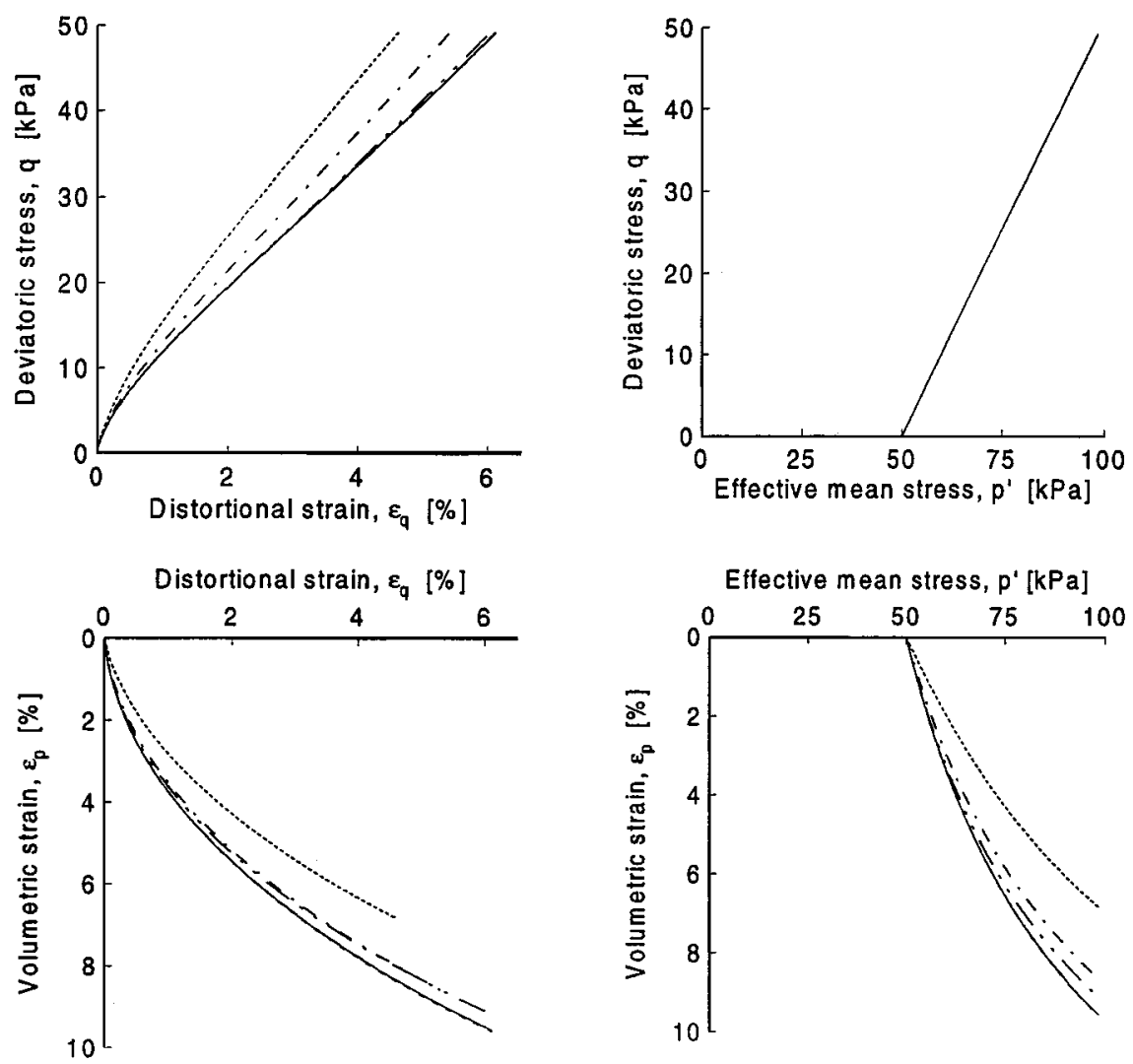

Number of objective function computations

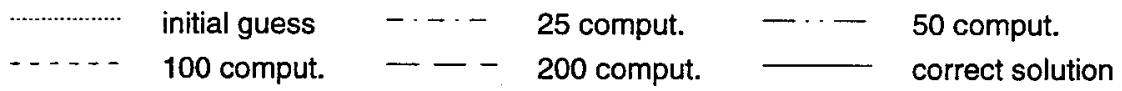

Figure 6. The simplex method-progresses during optimization for test 8 in Table VI.

Both the optimizations in Table II, leading to maximum values of $F_{\min }^{200}$, approached a local minimum which then was the reason for the poor result. Obviously, independent of the running time of an optimization, after a local minimum has been reached, the result will not be improved. For these optimizations, the initial step length used in the Rosenbrock method was $e=0.909$ and the size of the initial simplex used in the simplex method was $e=0.0197$. In Rosenbrock's method, the local minimum $F_{\text {min }}=96.6$ per cent was reached after 177 computations of the objective function. In the simplex method, it was possible to obtain a slight improvement after the 200 computations of the objective function in Table II and the local minimum $F_{\min }=69.1$ per cent was reached after 456 computations of the objective function.

Based on the observations discussed in this section, it can be concluded that the numerical performance of the optimization routine presented is good enough for finding a global minimum 
Table V. The simplex method-optimization variables.

\begin{tabular}{|c|c|c|c|c|c|c|}
\hline \multirow{2}{*}{$\begin{array}{l}\text { Number of } \\
\text { computations } \\
\text { of } F_{\text {comb }}\end{array}$} & \multirow{2}{*}{$\begin{array}{l}F_{\min } \\
(\%)\end{array}$} & \multicolumn{5}{|c|}{ Optimization variables } \\
\hline & & $\lambda^{*}$ & $D$ & $M$ & $N$ & $e_{r}$ \\
\hline 0 (initial) & 341.519 & 0.1 & 0.5 & 1.0 & 1.0 & 0.9 \\
\hline 25 & 80.6335 & 0.169515 & 1.33049 & 1.17982 & 0.998580 & 0.679243 \\
\hline 50 & 34.9594 & 0.195925 & 1.56445 & 1.19343 & 0.889186 & 0.637467 \\
\hline 75 & 9.78201 & 0.199244 & 1.52354 & 1.19214 & 0.811559 & 0.647814 \\
\hline 100 & 3.36355 & 0.199189 & 1.47976 & 1.20126 & 0.802552 & 0.651369 \\
\hline 150 & 0.276822 & 0.200036 & 1.49951 & 1.20013 & 0.799252 & 0.650080 \\
\hline 200 & 0.0311695 & 0.199990 & 1.50001 & 1.19999 & 0.799952 & 0.649999 \\
\hline 300 & 0.00415978 & 0.199998 & 1.49999 & 1.20000 & 0.799939 & 0.650001 \\
\hline 400 & 0.00410203 & 0.199998 & 1.49999 & 1.20000 & 0.799940 & 0.650001 \\
\hline 500 & 0.00410203 & 0.199998 & 1.49999 & 1.20000 & 0.799940 & 0.650001 \\
\hline 1000 & 0.00410203 & 0.199998 & 1.49999 & 1.20000 & 0.799940 & 0.650001 \\
\hline
\end{tabular}

with a much greater precision than is normally required in practise, if the optimization just continues a sufficiently long time and no local minimum is reached during the process. If there is reason to suspect that a local minimum has been reached, as a first attempt it is easy to try to restart the optimization with a new $e$-value and/or different search strategy.

It is also desirable that an optimization program should be fast, in order to limit the computational costs. Here, the constitutive simulations involved in the computation of the objective function require most of the computation time. Therefore a certain number of objective function computations demands approximately the same time for both the search strategies. For the large optimization problem analysed here, it takes in average $4.1 \mathrm{~s}$ between each objective function computation, when the problem runs on a PC with a $166 \mathrm{MHz}$ pentium processor and $64 \mathrm{Mb}$ RAM. This information might give an idea of how fast the presented optimization routine is.

\subsection{Optimization against simulated experimental result with randomly generated perturbations}

The objective of the following study is to examine the behaviour of the optimization routine when the simulated experimental result, in the previous section, is subjected to perturbations of different magnitude. As in Section 5.1, optimizations with the Rosenbrock and the simplex method have been performed against the large optimization problem constructed from tests no. 1 to 10 in Table I. In addition, optimizations against a smaller problem have been performed. This problem has been constructed from tests no. 4, 6 and 8 in Table I, in the same manner as the larger problem.

Perturbations have been introduced on the response parameters of the simulated experimental result. This has been done in all discrete points $z_{i}$, by randomly generate a new value $\tilde{z}_{i}$ inside the interval represented by the limits $z_{i}(1 \pm \psi)$ where the value of $\psi$ dictates the magnitude of the maximum perturbation. The pore pressure has been treated as a response parameter in the case of undrained behaviour. The magnitudes of maximum perturbation that have been examined are $\psi=0.25,0.50,0.75,1,2,3,5,7,10,15,20,25,30,35$ and 40 per cent. 


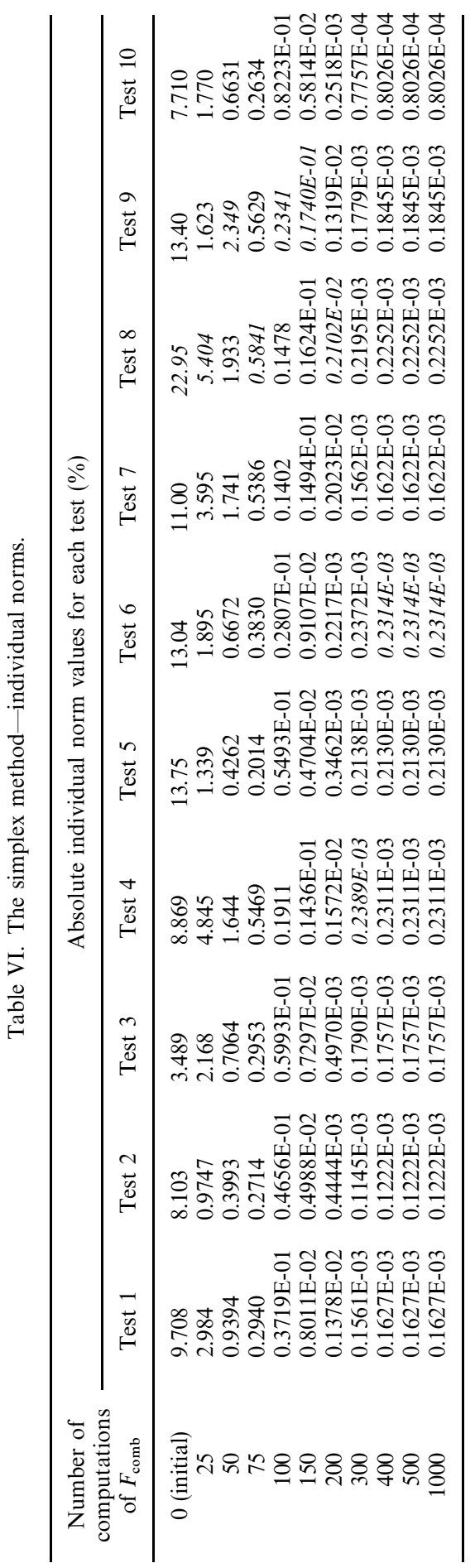


In Figure 7, for each optimization variable, the optimal values found have been plotted vs the examined magnitudes of maximum perturbation in a semi-logarithmic scale. For all the values of $\psi$, the optimal set of parameters has been determined out from 100 optimizations, with 200 computations of the objective function and a randomly chosen $e$-value in each optimization, respectively.

The most important conclusion from this study is that the optimization routine is able to find a minimum even though the experimental result is substantially perturbed. Furthermore, for small random errors in the experimental result, of a magnitude that is likely in experimental tests with a good equipment, e.g. $\psi<5$ per cent, the set of optimization variables in Figure 7 obtains values very close to the global minimum associated with $\psi=0$. This indicates that it is not necessary to make any fine adjustments to the experimental result before an optimization is performed.

It is seen that larger deviations from the global minimum associated with $\psi=0$ are gradually obtained when the value of $\psi$ is increasing. No clear trend for any parameter to deviate in a certain direction is noticed. The values seems to oscillate around the global minimum of the undisturbed experimental result.

According to the laws of probability, the greater the number of tests involved in an optimization are the more likely for random errors to be compensating. Therefore, it is reasonable to expect larger deviations in an earlier stage for the optimizations against tests no. 4, 6 and 8 compared to optimizations against tests no. 1-10. However, this is not an effect that appears distinctly in Figure 7, except perhaps for the parameters $\lambda^{*}, D$ and $M$ when $\psi=20$ per cent.

Only small differences in the result are displayed in a comparison between the Rosenbrock and the simplex method. The simplex method gives, however, in general a slightly lesser deviation from the global minimum associated with $\psi=0$, probably because it is a more effective search strategy for this example which has been pointed out before.

\subsection{Optimization against performed triaxial tests}

In this section, the application of the optimization program to performed laboratory tests is demonstrated. The tests used are three true triaxial tests reported by Wood and Wroth [15]. These tests were performed in order to study the validity of some of the assumptions made in interpreting the results from undrained pressuremeter expansion tests. Hence, the same strain path was applied to the soil specimen in the true triaxial apparatus as is believed to be followed by the elements of soil around a pressuremeter. The laboratory tests were performed on a slurry of Spestone kaolin clay and water, laboratory prepared to different overconsolidation ratios.

In the tests by Wood and Wroth [15] the stress/strain history of typical soil deposits were initially obtained in the true triaxial apparatus. In Table VII, the states for these histories of one-dimensional consolidation and unloading (no lateral displacement) are prescribed for the

three tests $\mathrm{K} 2-\mathrm{K} 4$. The stresses $\sigma_{1_{\max }}$ and $p_{\max }^{\prime}$ are the maximum effective stress in the vertical direction (1-direction of the apparatus) and the maximum effective mean stress reached in one-dimensional consolidation, respectively, whereas $\sigma_{1_{0}}$ and $p_{0}^{\prime}$ are the values of the corresponding stresses after unloading. It should be noticed that the values of $K_{0}$ in Table VII, the ratio between the horizontal and the vertical effective stress, are presented at the state of normal consolidation, i.e. after the one-dimensional consolidation phase. In Table VII, it is then seen that tests K2 and K3 represent an overconsolidated soil (where test no. K3 has a higher overconsolidation ratio than test no. K2) while test no. K4 represents a normally consolidated soil. 

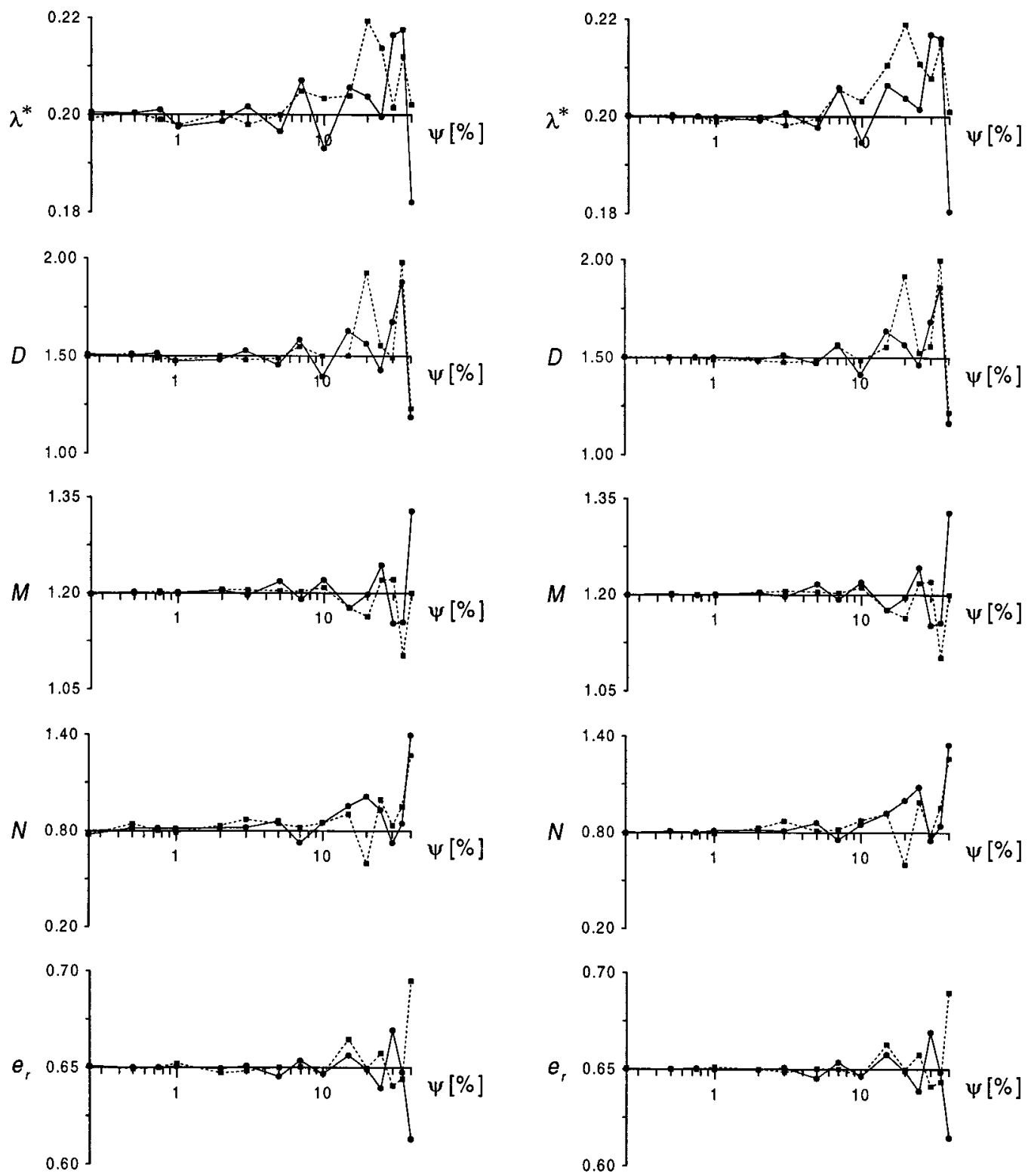

(a)

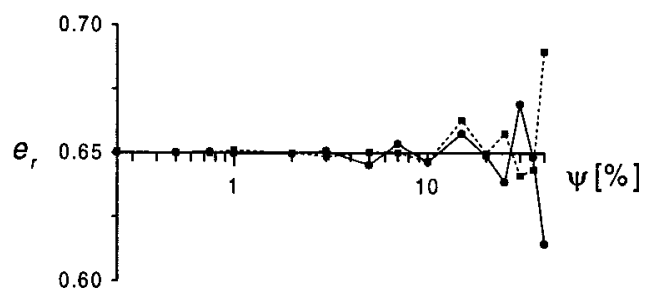

(b)

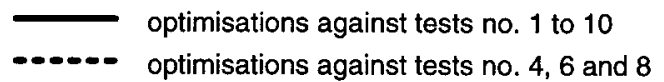

Figure 7. Optimization variables vs magnitude of maximum perturbation: (a) The Rosenbrock method; (b) the simplex method. 
Table VII. State after the one-dimensional consolidation and unloading phase.

\begin{tabular}{ccccccc}
\hline $\begin{array}{c}\text { Test } \\
\text { number }\end{array}$ & $\begin{array}{c}\text { Specific } \\
\text { volume }\end{array}$ & $\begin{array}{c}\sigma_{1_{\max }} \\
(\mathrm{kPa})\end{array}$ & $\sigma_{1_{\max }} / \sigma_{1_{0}}$ & $\begin{array}{c}p_{\max }^{\prime} \\
(\mathrm{kPa})\end{array}$ & $p_{\max }^{\prime} / p_{0}^{\prime}$ & $K_{0}^{\mathrm{NC}}$ \\
\hline $\mathrm{K} 2$ & 2.280 & 483 & 2.50 & 380 & 2.10 & 0.68 \\
$\mathrm{~K} 3$ & 2.165 & 934 & 5.46 & 774 & 4.31 & 0.75 \\
$\mathrm{~K} 4$ & 2.299 & 351 & 1.00 & 282 & 1.00 & 0.71 \\
\hline
\end{tabular}

In an undrained pressuremeter expansion test, it is normally presumed that the soil is deformed in plane strain with the vertical direction as the direction in which no strain occur. Further, the soil is compressed in the radial direction, and to fulfil the incompressibility constraint a balancing circumferential extension has to be presumed. If pore pressures are measured during an undrained pressuremeter expansion test the effective stresses can be deduced out from the interpreted total stresses. By applying the deformation mode

$$
\varepsilon_{2}=-\varepsilon_{3}, \quad \varepsilon_{1}=0, \quad \varepsilon_{2}>0
$$

to the cubical sample in a true triaxial apparatus under drained conditions, the effective stresses measured could be compared to the effective stresses obtained from the pressuremeter test. Thereby, conclusions about the validity of the assumptions made for interpreting the pressuremeter results could be drawn. In the paper by Wood and Wroth [15], samples K2-K4 were sheared to failure with the strain rates $\dot{\varepsilon}_{1}, \dot{\varepsilon}_{2}, \dot{\varepsilon}_{3}=0,0.25,-0.25$ per cent/h according to the deformation mode in Equation (30). The suffixes used refer to the fixed axes of the true triaxial apparatus. The strain rates were reversed a few times during these laboratory tests, but it is only results from this first shearing to failure that will be used here as a basis for optimization.

Two different constitutive models were chosen for the optimizations: the well-known modified cam clay model (MCC) and the anisotropic cam clay model (ACC) described by Runesson and Axelsson [16] and $\mathrm{Yu}$ [17]. In a discussion about models that could be suitable for prediction of this kind of tests, carried out by Wood in Reference [18], it was expected that a model like MCC would not be particularly successful and a model like ACC was suggested to be more appropriate. The anisotropic cam clay model is based on the modified cam clay concept but the $K_{0}^{\mathrm{NC}}$-line in the effective stress space is used as a symmetry line for the yield surface in contrast to the modified cam clay model where the yield surface is centred on the stress space diagonal. In addition, in the ACC model, the destruction of the initial $K_{0}$-anisotropy can be modelled with a rotational hardening/softening law and deviatoric anisotropy can be simulated by distorting the yield locus in the deviatoric plane. Seven parameters are included in the ACC model, i.e.

$\kappa$ the slope of the unloading-reloading line in the $v-\ln p^{\prime}$ plane, where $v$ is the specific volume and $p^{\prime}$ the effective mean stress

$v^{\prime}$ Poisson's ratio

$\lambda \quad$ the slope of the $K_{0}$-compression line in the $v-\ln p^{\prime}$ plane

$C$ parameter for rotational hardening/softening

$M^{f}$ the slope of the failure line a $p^{\prime}-q$ plane

$k$ the intercept of the failure line

a parameter for deviatoric anisotropy 
The parameters $\kappa, v^{\prime}$ and $\lambda$ are utilized also in the MCC model, together with the slope of the critical state line $(M)$.

Individual optimizations (one test at a time) and simultaneous optimizations (all tests at the same time) have been performed against tests K2-K4, with both the anisotropic cam clay and the modified cam clay model. All parameters in the models will have an influence on the predicted results for these tests.

Experimentally, it is difficult to exactly follow the intended deformation mode. Therefore, a linear variation of the principal strains, between the discrete states obtained in the triaxial tests, have been assumed in the constitutive simulations. The best agreements between experiments and predictions obtained by optimization are shown by plots in $q-\varepsilon_{q}, q-p^{\prime}$ and $\pi$-planes, in Figure 8 for the individual optimizations and in Figure 9 for the simultaneous optimizations. The optimal set of model parameters and the value of the maximum norm ( $F_{\max }$ in Equation (12) utilizing $E_{\text {abs }}$ in Equation (7) with $w=1.0$ ) corresponding to these optimizations are shown in Table VIII for the ACC model and in Table IX for the MCC model.

The curve plots in Figures 8 and 9 as well as a comparison of the maximum norm values in Tables VIII and IX are everywhere showing a better agreement to the laboratory tests for the ACC model than for the MCC model. For the ACC model, a 'reasonably good' agreement have been achieved in all optimizations. With the MCC model, the agreement can be said to be 'reasonably good' only for the individual optimization against test no. K4. This is however to be expected, since the MCC model is historically founded mostly on data from conventional triaxial tests on samples without pronounced $K_{0}$-anisotropy. It should be remembered that, even if the model is not able to predict the soil behaviour properly, the optimization process tries to find the best possible agreement and sometimes, as a consequence, delivers strange parameter values. As could be expected, better agreement was obtained for the individual optimizations compared to the simultaneous optimizations. But for the ACC model, it is promising to see that a reasonable agreement has been obtained also in the simultaneous optimization. In the simultaneous optimizations the combined norm in Equation (13) together with the absolute norm (without weighting) in Equation (7) have been used. However, maximum norm values are presented in Tables VIII and IX to facilitate comparisons with norm values of the individual optimizations. The set of norm values obtained for the simultaneous optimizations in Tables VIII and IX, respectively, were $F_{\text {comb }}=42.2$ per cent, $E_{\mathrm{abs}}^{\mathrm{K} 2}=5.82$ per cent, $E_{\mathrm{abs}}^{\mathrm{K} 3}=7.55$ per cent, $E_{\mathrm{abs}}^{\mathrm{K} 4}=6.13$ per cent and $F_{\text {comb }}=94.9$ per cent, $E_{\mathrm{abs}}^{\mathrm{K} 2}=16.2$ per cent, $E_{\mathrm{abs}}^{\mathrm{K} 3}=14.4$ per cent, $E_{\mathrm{abs}}^{\mathrm{K} 4}=15.8$ per cent. It is seen that the values of $F_{\max }$ presented for the simultaneous optimizations in Tables VIII and IX were obtained for tests no. K3 and K2, respectively. For both the models, the individual optimization against test no. $\mathrm{K} 4$ gave the best result while the optimization against test no. K3 gave the worst result. It seems that both the applied models have more difficulties in predicting this kind of tests for higher overconsolidation ratios.

Comparisons between the values of the optimal set of parameters, obtained in the different optimizations, show that they vary rather much. For the individual optimizations, this variation clearly appears, anyway the prediction curves (at least for the ACC model) are located quite close to the experimental curves. This could be explained by a co-operation of the parameters, i.e. there exist more than one set of parameters that gives good agreement, but it could also indicate shortcomings of the model. In the ACC model, some co-operation between the parameters could be expected, e.g. between $M^{f}$ and $k$. An unexpected result, most likely caused by co-operation between the parameters, was that the parameters $\lambda$ and $a$ in the simultaneous optimization with the ACC model did not end up in-between the lowest and the highest values obtained in the 

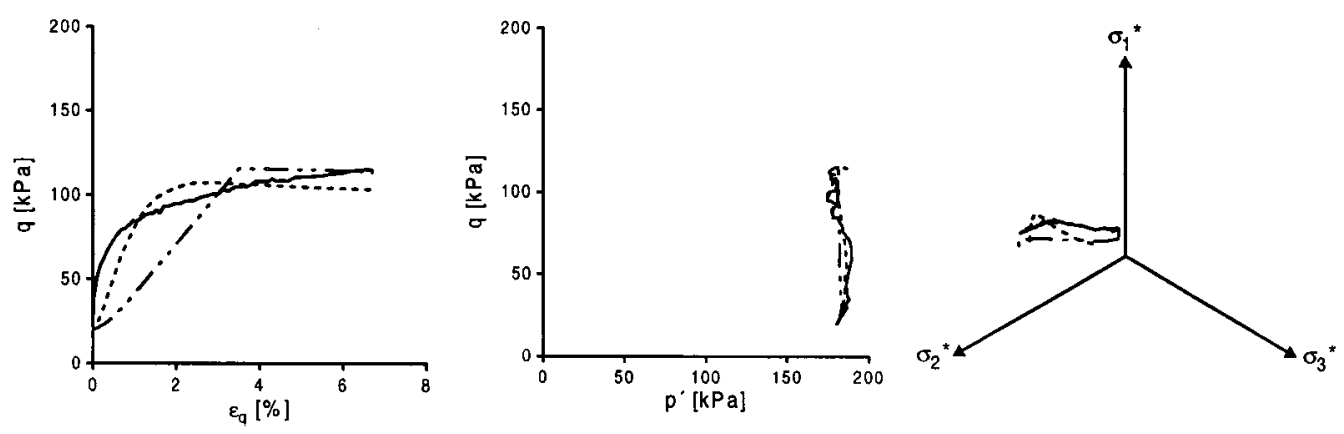

(a)
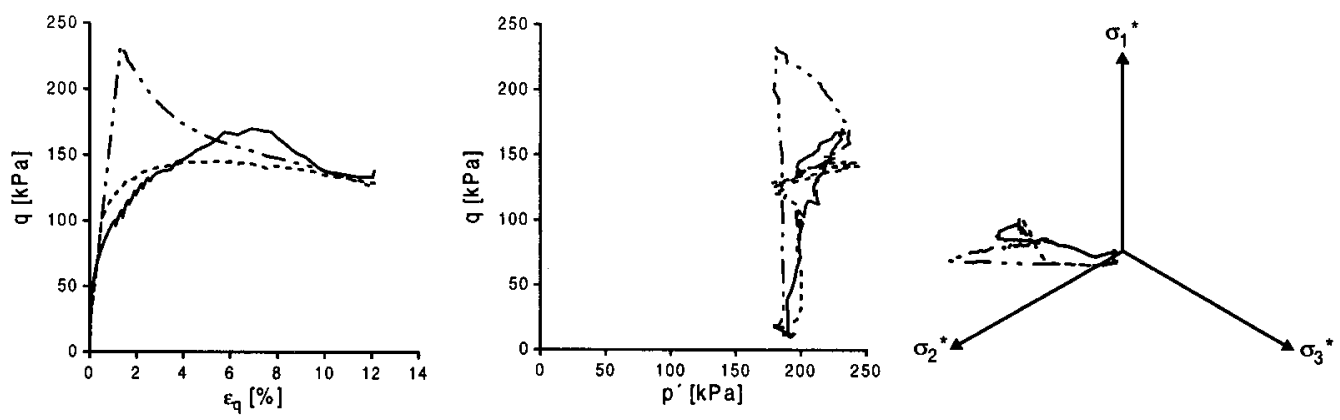

(b)
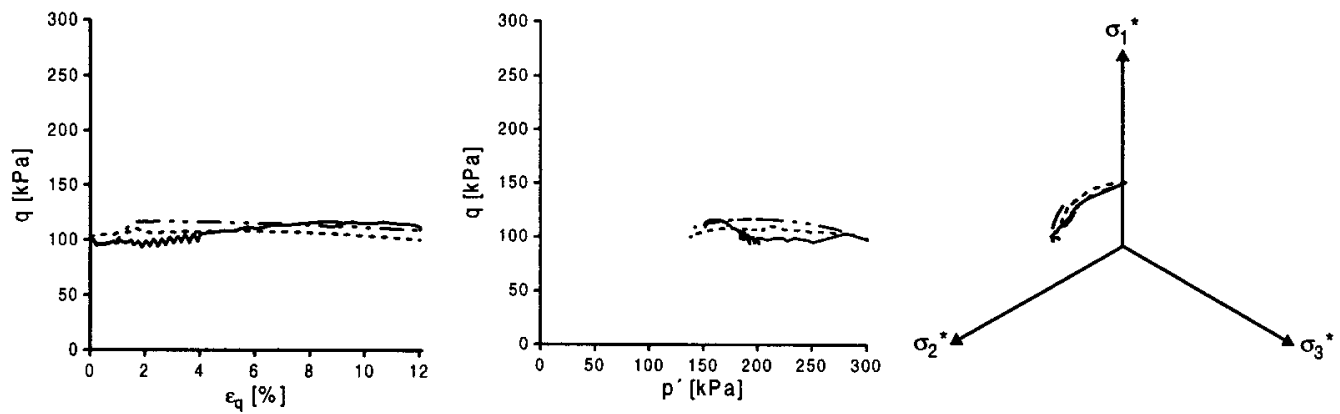

(c)

Figure 8. Optimization against individual tests: (a) test K2; (b) test K3; (c) test K4.

individual optimizations. Further, the soil will probably not obey the non-linear isotropic elastic law used in the models, so the elastic parameters $\kappa$ and $v^{\prime}$ are loosing their physical interpretation and will be acting more like curve fitting parameters. The parameter variation observed, reveals the danger in believing that parameters evaluated from a certain test can successfully be used for 

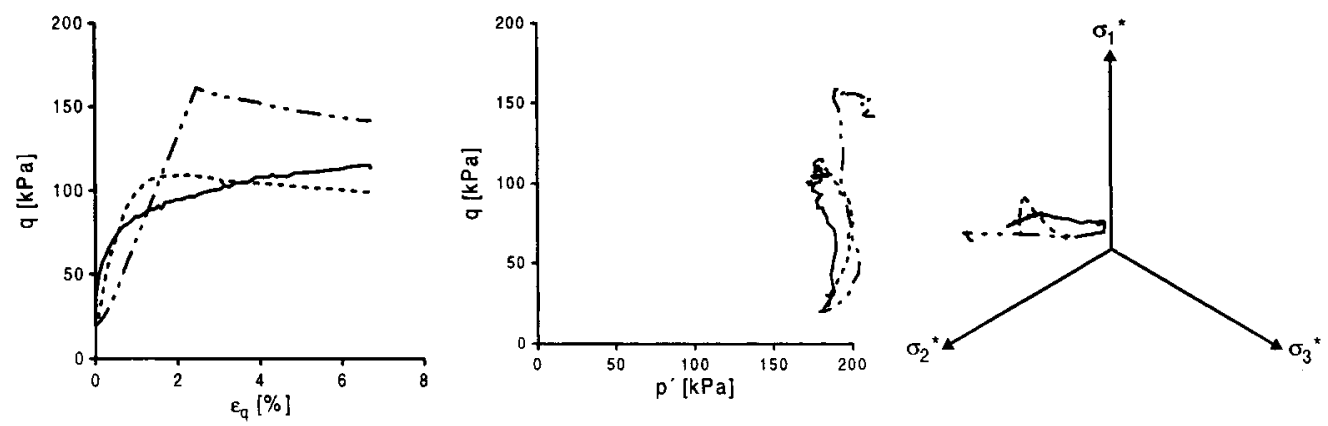

(a)
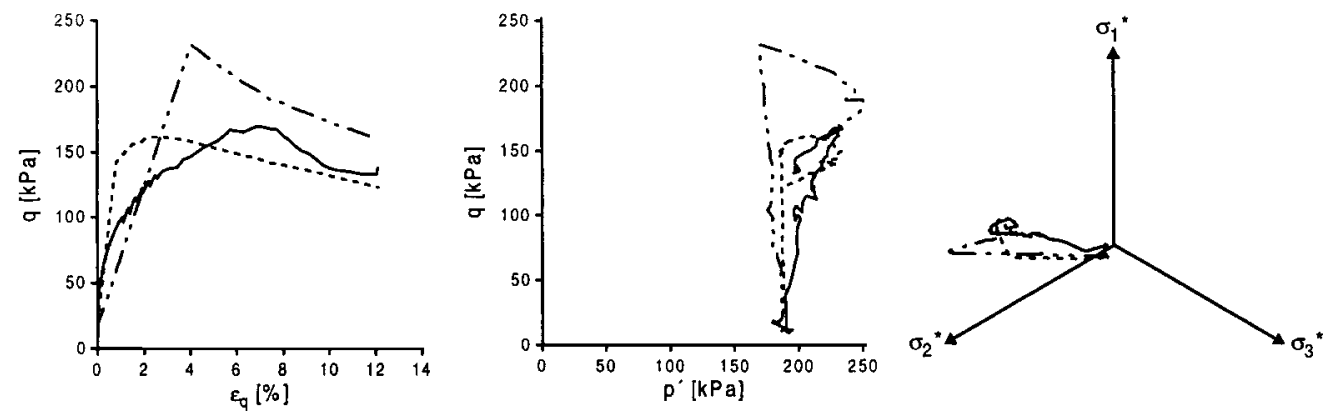

(b)
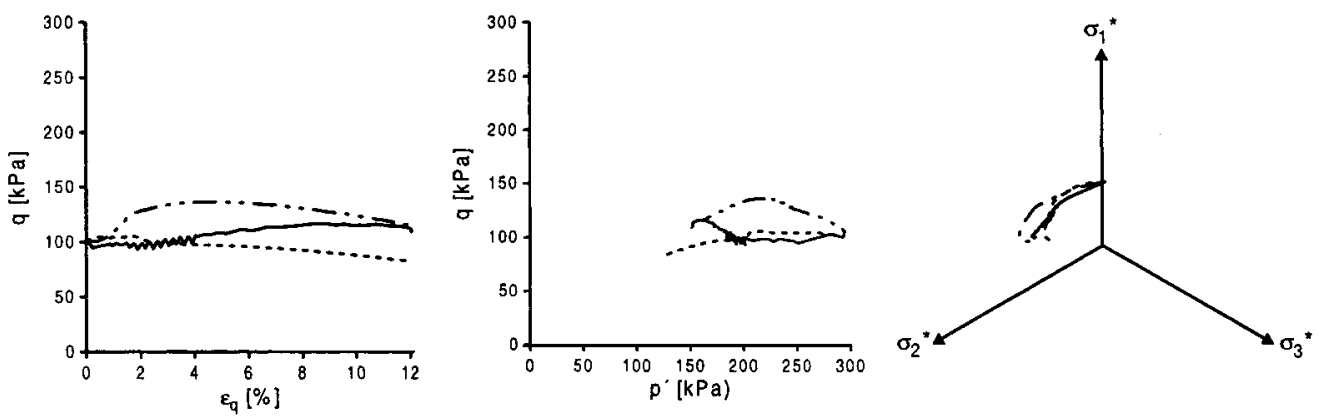

(c)

Figure 9. Simultaneous optimization against the tests K2, K3 and K4: (a) test K2; (b) test K3; (c) test K4. other stress/strain paths, in e.g. the finite element method. If a constitutive model is impaired by such a weakness, and no better model could be found, parameters might be evaluated from many qualitatively different types of tests, e.g. by an optimization program utilizing simultaneous optimization of all appropriate tests. 
Table VIII. The optimal set of parameters-anisotropic cam clay.

\begin{tabular}{|c|c|c|c|c|c|c|c|c|}
\hline \multirow{2}{*}{$\begin{array}{l}\text { Optimization } \\
\text { against test }\end{array}$} & \multirow{2}{*}{$\begin{array}{c}F_{\max } \\
\text { Equation }(12) \\
(\%)\end{array}$} & \multicolumn{7}{|c|}{ Optimization variables } \\
\hline & & $\kappa$ & $v^{\prime}$ & $\lambda$ & C & $M^{f}$ & $k$ & $a$ \\
\hline $\mathrm{K} 2$ & 3.99 & 0.164 & 0.0813 & 0.400 & 38.5 & 0.510 & 9.97 & 1.00 \\
\hline K3 & 6.90 & 0.0184 & 0.391 & 0.183 & 15.2 & 0.442 & 16.6 & 0.649 \\
\hline K4 & 3.81 & 0.0598 & 0.00264 & 0.185 & 22.5 & 0.640 & 0.0700 & 0.950 \\
\hline $\mathrm{K} 2, \mathrm{~K} 3, \mathrm{~K} 4$ & 7.55 & 0.0420 & 0.265 & 0.101 & 27.9 & 0.590 & 8.27 & 0.585 \\
\hline
\end{tabular}

Table IX. The optimal set of parameters - modified cam clay.

\begin{tabular}{lccccc}
\hline $\begin{array}{l}\text { Optimization } \\
\text { against test }\end{array}$ & $\begin{array}{c}F_{\max } \\
\text { Equation } \\
(\%)\end{array}$ & \multicolumn{4}{c}{ Optimization variables } \\
\cline { 3 - 6 } & 8.22 & 0.400 & $v^{\prime}$ & $\lambda$ & $M$ \\
\hline K2 & 9.55 & 0.0510 & 0.132 & 0.800 & 0.231 \\
K3 & 4.97 & 0.0175 & 0.467 & 0.0814 & 0.618 \\
K4 & 16.2 & 0.0480 & 0.396 & 0.589 & 0.594 \\
K2, K3, K4 & & & & 0.104 & 0.626 \\
\hline
\end{tabular}

In practise, the solution of the optimization problem might not be the only information requested. The reliability of the estimated parameters as well as the sensitivity of the solution to changes in these parameters can also be of interest. An attractive feature of optimization techniques is the possibility to perform the reliability and the sensitivity analysis more or less simultaneously when the minimum of the objective function has been reached. Mathematically, sufficient conditions for a local minimum are a gradient vector $\nabla F(\mathbf{x})$ with all of the elements equal to zero and a Hessian matrix $\nabla^{2} F(\mathbf{x})$ that is positive definite, see e.g. Reference [19]. With the Hessian matrix, the curvature of $F(\mathbf{x})$ can be calculated in any direction and the curvature in each co-ordinate direction is given by $\partial^{2} F /\left(\partial x^{j} \partial x^{j}\right)$. The curvature is a measure of the sensitivity of the solution to small parameter changes. If possible, sensitivity should also be related to how accurate the parameters can be determined experimentally. Derivatives of the type of objective function proposed in this paper have to be calculated numerically. Even if the smoothness properties of the objective function are unknown, the conditions for a local minimum above can, presumably, be numerically evaluated without any problem in most cases.

Studies of reliability and sensitivity were performed on the optimization result obtained with the ACC model in Table VIII. As a first step, second partial derivatives of $F$ in the co-ordinate directions were computed with the expression $\partial^{2} F /\left(\partial x^{j} \partial x^{j}\right)=\left[F\left(x^{j}+h\right)-2 F\left(x^{j}\right)+F\left(x^{j}-h\right)\right] / h^{2}$ where $h=5 \times 10^{-4}$ was chosen. The values of $F\left(x^{j}+h\right)$ and $F\left(x^{j}-h\right)$ turned out to be larger than, or equal to, $F\left(x^{j}\right)$ for both the individual optimizations and the simultaneous optimization. This proves that at least stationary points were reached (no Hessian matrices were computed). A variation of the magnitude of the curvature in the co-ordinate directions were shown in each test, respectively, but no pronounced trend for any of the parameters to be more sensitive or 
insensitive than the others were displayed in a comparison between the tests. A substantial variation of the curvature magnitude was also noticed amongst the tests, e.g. test no. K3 obtained curvature magnitudes about 4000 times higher than those of test no. K2. Thereafter, properties of $F(\mathbf{x})$ were examined at small distances from the minima in Table VIII, by gradually computing $F\left(1.05 x^{j}\right)$ and $F\left(0.95 x^{j}\right)$ for each parameter, keeping the other parameters constant. The parameter $M^{f}$ distinctly appeared to be the most sensitive parameter in this study and it looked as if $k$ was the most insensitive parameter.

\section{CONCLUDING REMARKS}

An efficient and user-friendly optimization routine, of the type presented here, should be a useful tool for both researchers and practising engineers dealing with advanced soil mechanics, especially when the constitutive models tend to be more complicated as the knowledge about soil behaviour increases. Such a program could be used for the evaluation of established as well as new constitutive models, but the widest area of application is in the process of choosing adequate constitutive models and the determination of their parameter values for the application to geotechnical problems using numerical methods of solution, e.g. FEM, FEM/BEM or FDM. The need for an optimization routine in connection to such programs becomes obvious, since several alternative constitutive models normally are implemented to meet the requirement of simulating different types of soil. Further, with some smaller modifications the proposed optimization routine could be used for other materials than soils as well.

In addition to parameter optimization at the constitutive level (as in a Constitutive Driver), the proposed optimization routine might also be used for optimization at the entire sample level where tests are simulated as boundary value problems, with e.g. the finite element method. Then, it is no longer necessary to neglect boundary effects that can have substantial influences on the obtained result, see e.g. Reference [20] where effects of end restraint and strain rate in triaxial testing is discussed. However, optimization at the sample level can be expected to be quite computationally expensive. It is, therefore, advantageous to optimize as much as possible at the constitutive level before an optimization at the same level starts.

This paper is focused on the mathematical aspects of the optimization routine. However, if an optimization program should be practically useful, a lot of effort must be spend on creating a user-friendly software. In the optimization program discussed here a pre- and a post-processor are included. The input necessary to define the optimization problem are given in the preprocessor and could also be saved in a database. The database could then, in a later run, be read directly into the program. It is possible both to define additional tests to the database and delete existing tests from the database, inside the program. In the post-processor, it is possible to graphically interpret the agreement between experimental and predicted result in curve plots with different stress-strain invariants and state parameters. If a sufficient agreement has not been reached, a new optimization could be started from this stage without leaving the program. The restart could be performed with another search strategy, different $e$-value, a new set of optimization variables, new initial starting point, new lower and upper bounds, different objective function, different termination conditions, etc. All this is essential to make it easier to locate the global minimum. On the other hand, it is important to remind that even the best optimization program cannot overcome the shortcomings of a model and replace the engineering experience. 
Even though the 'best' optimization algorithm for the present application can only be determined by comparative testing of available methods, it has been found in the present study that the proposed optimization routine is general, easy to program and works well for optimization of model parameters in soil plasticity.

\section{ACKNOWLEDGEMENTS}

The authors would like to thank Prof. David Muir Wood at the University of Bristol, U.K. for providing the experimental results used in Section 5.3. Financial support from TFR (Swedish Research Council for Engineering Science) and SBUF (the Development Fund of the Swedish Construction Industry) is gratefully acknowledged. The second author would also like to express thanks to Swedish Building Research Council (BFR) for financial support to basic research in structural mechanics.

\section{APPENDIX A: EVALUATION OF THE STEP ADJUSTMENT PARAMETERS $\alpha$ AND $\beta$ IN ROSENBROCK'S METHOD}

The objective of this appendix is to find the most effective combination of values of the step adjustment parameters $\alpha$ and $\beta$, for use in the version of Rosenbrock's search algorithm implemented in the optimization program. In this context, an efficient algorithm is defined as an algorithm that advances as much as possible towards a minimum during a given computation time. The optimization problem defined above in Section 5.1, was regarded as a representative problem for the type of optimization discussed in this paper and was therefore chosen as the 'evaluation example' for this analysis.

The interval of interest for the contraction parameter, $0<\beta<1$, directly follows from the algorithm in Section 4.1 and it was considered to be sufficient to examine the expansion parameter inside the interval $1<\alpha<5$. The main idea was to perform repeated optimizations on the 'evaluation example' where the initial step value $0<e<1$, the expansion parameter $\alpha$ and the contraction parameter $\beta$ were given randomly generated values inside their intervals according to certain patterns for each new optimization, in order to (out from the obtained result) confine the study to smaller intervals until $\alpha$ and $\beta$ could be fixed.

As a first step a set of optimizations were performed where both $e$ and $\beta$ were randomly generated for each new optimization while $\alpha$ was randomly generated gradually at intervals of 25 optimizations and kept constant in-between. The obtained result is shown in Figure A1 where, for each optimization, the smallest objective function value $F_{\min }$, after 200 computations of the objective function in step 3.3 in the algorithm, has been plotted against the contraction parameter $\beta$. It is seen that, independent of the value of $\alpha$, a limited interval approximately between $0.6<\beta<0.9$ exists where the values of the objective function $F_{\min }^{200}$ in general are smaller and less spread than in adjacent areas. Further, this interval could be subdivided into two subintervals, $0.6<\beta<0.8$ and $0.8<\beta<0.9$. If these subintervals are compared to each other, it is seen that the values of $F_{\min }^{200}$ are more widely spread in the first subinterval where both the largest and smallest values of $F_{\min }^{200}$ are found. It was decided to fix the contraction parameter to the intermediate point $\beta=0.8$, where the scattering starts to decrease at the same time as the values of $F_{\min }^{200}$ are comparatively low.

To evaluate the influence of $\alpha, 400$ optimizations were performed with the value of $\beta$ fixed to 0.8 and the values of $0<e<1$ and $1<\alpha<5$ randomly generated inside their respective intervals for 


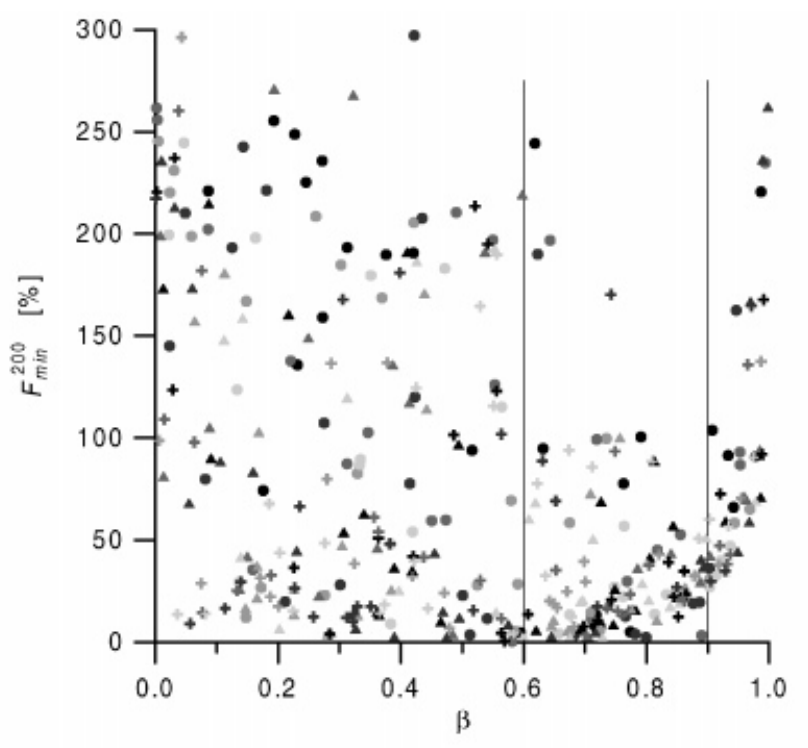

$\begin{array}{cccccc}\alpha=1.019 & \Delta & \alpha=2.361 & + & \alpha=3.035 \\ \alpha & \alpha=1.292 & \Delta & \alpha=2.425 & + & \alpha=3.281 \\ \alpha=1.314 & \Delta & \alpha=2.625 & + & \alpha=3.497 \\ \alpha=1.491 & \Delta & \alpha=2.716 & + & \alpha=3.948 \\ \alpha=2.027 & \Delta & \alpha=2.843 & + & \alpha=4.903\end{array}$

Figure A1. Objective function value vs contraction parameters $\beta$.

each new optimization. For these optimizations, the smallest value of the objective function $F_{\min }$, after 200 computations of the objective function in step 3.3 in the algorithm, is plotted against the expansion parameter $\alpha$ in Figure A2. It is seen that the result is quite scattered, but almost every optimization resulted in a value of $F_{\text {min }}^{200}$ below 100 per cent. This is another evidence that the fixation of $\beta$ to 0.8 improves the behaviour of the algorithm. A clear tendency for the smallest obtained values of $F_{\min }^{200}$ to increase with higher values of $\alpha$, for $\alpha \geqslant 1.3$, is however shown in Figure A2. The interval $1.3 \leqslant \alpha \leqslant 2.0$ was considered to be the one of most interest. This interval, where both small values of $F_{\min }^{200}$ and relatively less scattering was obtained, was closer analysed and the result is shown in Table A1 where the average value, the standard deviation, the minimum and the maximum obtained values of $F_{\min }^{200}$ computed out from 100 optimizations for each case of $\alpha$ are presented. Because both the smallest average value and the smallest standard deviation were obtained for the case $\alpha=1.9$, it was decided to fix $\alpha$ to 1.9.

\section{APPENDIX B: EVALUATION OF THE REFLECTION, EXPANSION AND CONTRACTION COEFFICIENTS IN THE SIMPLEX METHOD}

In this appendix, the most effective combination of the reflection, expansion and contraction coefficients is searched for. The study is carried out in a similar way as in Appendix A, with the 


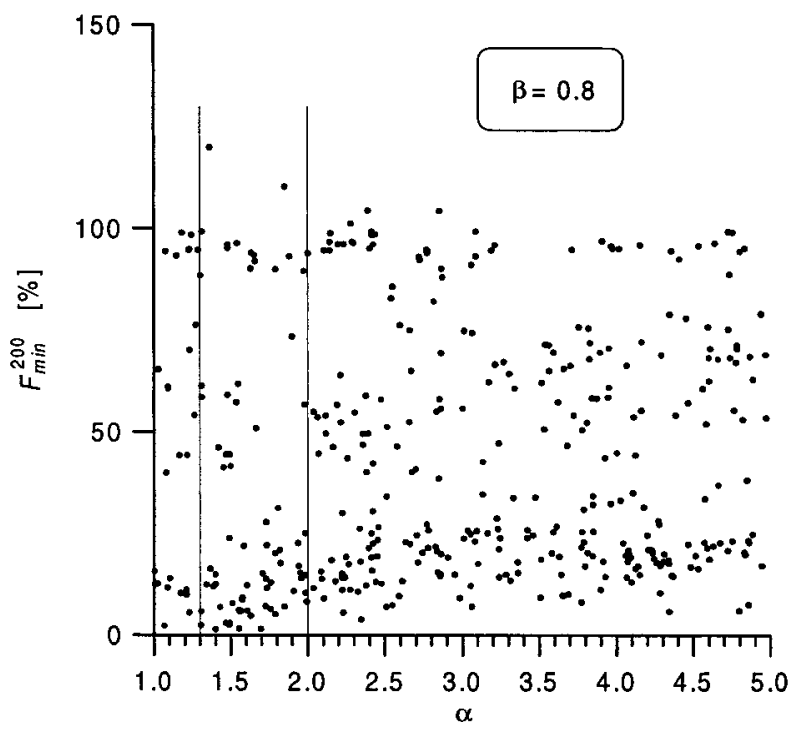

Figure A2. Objective function value vs expansion parameter $\alpha$.

Table A1. A closer analysis of $F_{\min }^{200}$ in the interval $1.3 \leqslant \alpha \leqslant 2.0$ with $\beta=0.8$.

\begin{tabular}{lcccccccc}
\hline Case $\backslash \alpha$ & 1.3 & 1.4 & 1.5 & 1.6 & 1.7 & 1.8 & 1.9 & 2.0 \\
\hline $\begin{array}{l}\text { Average value } \\
F_{\min }^{200}(\%)\end{array}$ & 47.5 & 42.2 & 41.5 & 41.8 & 44.4 & 42.0 & 39.6 & 42.9 \\
$\begin{array}{l}\text { Std. deviation } \\
F_{\min }^{200}(\%)\end{array}$ & 37.4 & 34.4 & 35.9 & 34.4 & 32.0 & 31.7 & 30.2 & 36.2 \\
$\begin{array}{l}\text { Min. value } \\
F_{\min }^{200}(\%)\end{array}$ & 1.35 & 0.87 & 0.59 & 2.01 & 1.95 & 0.54 & 1.34 & 2.44 \\
$\begin{array}{l}\text { Max. value } \\
F_{\min }^{200}(\%)\end{array}$ & 150.5 & 125.1 & 138.3 & 141.8 & 126.0 & 95.6 & 96.6 & 168.6 \\
\hline
\end{tabular}

optimization problem defined in Section 5.1 as the 'evaluation example'. Suitable intervals to search within was considered to be $0<\gamma<2$ for the reflection coefficient, $1<\zeta<5$ for the expansion coefficient and $0<\eta<1$ for the contraction coefficient.

In a first attempt, 300 optimizations were performed in which the values of the coefficients $\gamma$, $\zeta$ and $\eta$ were randomly generated inside their respective interval for each new optimization. Additionally the scalar $0<e<1$, defining the size of the starting simplex, was also randomly generated inside its interval for each new optimization. In the following analysis, as a first step, it turned out to be favourable to try to fix the reflection coefficient. For each optimization, the smallest obtained value of the objective function after 200 computations $^{\dagger}$ of the objective function ${ }^{\dagger}$ To be able to directly compare with results from Rosenbrock's algorithm, the first objective function computation is
however not counted. 


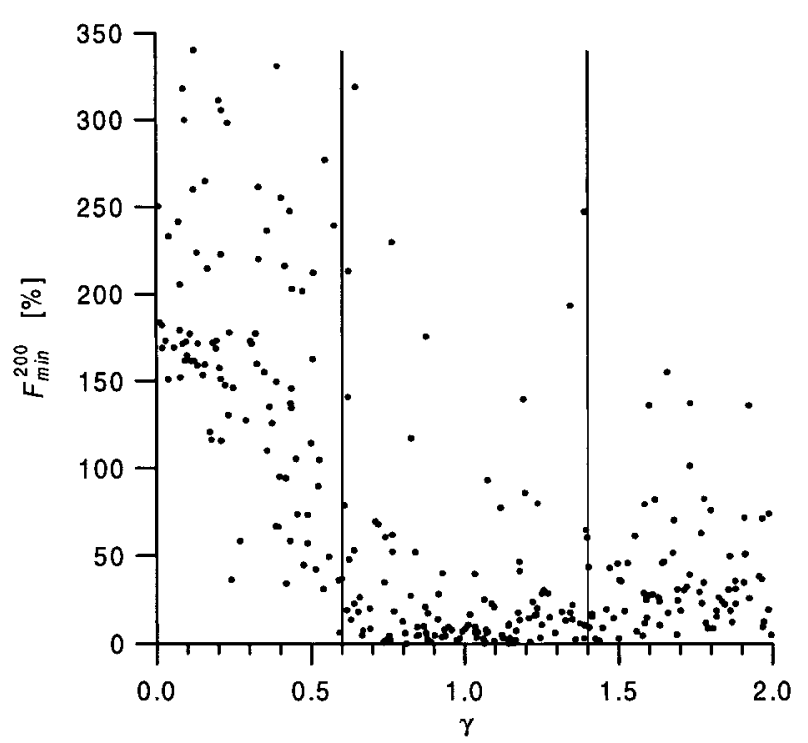

Figure B1. Objective function value vs reflection coefficient $\gamma$.

Table B1. A closer analysis of $F_{\min }^{200}$ in the interval $0.6 \leqslant \gamma \leqslant 1.4$.

\begin{tabular}{lccccc}
\hline Case $\backslash \gamma$ & 0.6 & 0.8 & 1.0 & 1.2 & 1.4 \\
\hline $\begin{array}{l}\text { Average value } \\
F_{\min }^{200}(\%)\end{array}$ & 58.1 & 27.7 & 25.4 & 32.3 & 37.7 \\
$\begin{array}{l}\text { Std. deviation } \\
F_{\min }^{200}(\%)\end{array}$ & 65.4 & 52.1 & 43.3 & 41.9 & 47.4 \\
$\begin{array}{l}\text { Min. value } \\
F_{\min }^{200}(\%)\end{array}$ & 0.17 & 0.008 & 0.015 & 0.27 & 0.37 \\
$\begin{array}{l}\text { Max. value } \\
F_{\min }^{200}(\%)\end{array}$ & 334.1 & 299.2 & 215.2 & 226.3 & 257.0 \\
\hline
\end{tabular}

in the algorithm was plotted against the reflection coefficient in Figure B1. It is seen that the smallest and least spread values of $F_{\min }^{200}$ were obtained in the interval $0.6 \leqslant \gamma \leqslant 1.4$, which motivated a closer analysis inside this interval. Based on 200 optimizations for each fixed value of the reflection coefficient, $\gamma=0.6,0.8,1.0,1.2$ and 1.4 , with the other quantities $\zeta, \eta$ and $e$ randomly generated as before, the average value, the standard deviation, the minimum value and the maximum value of $F_{\min }^{200}$ are shown in Table B1. Since the smallest average value were obtained for $\gamma=1.0$ as well as the second best standard deviation 43.3 per cent (next to 41.9 per cent for $\gamma=1.2$ ), it was decided to fix the reflection coefficient to $\gamma=1.0$.

As a next step, additional optimizations were performed with $\gamma$ fixed to 1.0 and $\zeta, \eta$ and $e$ randomly generated inside their intervals until a base of 500 optimizations were obtained. 


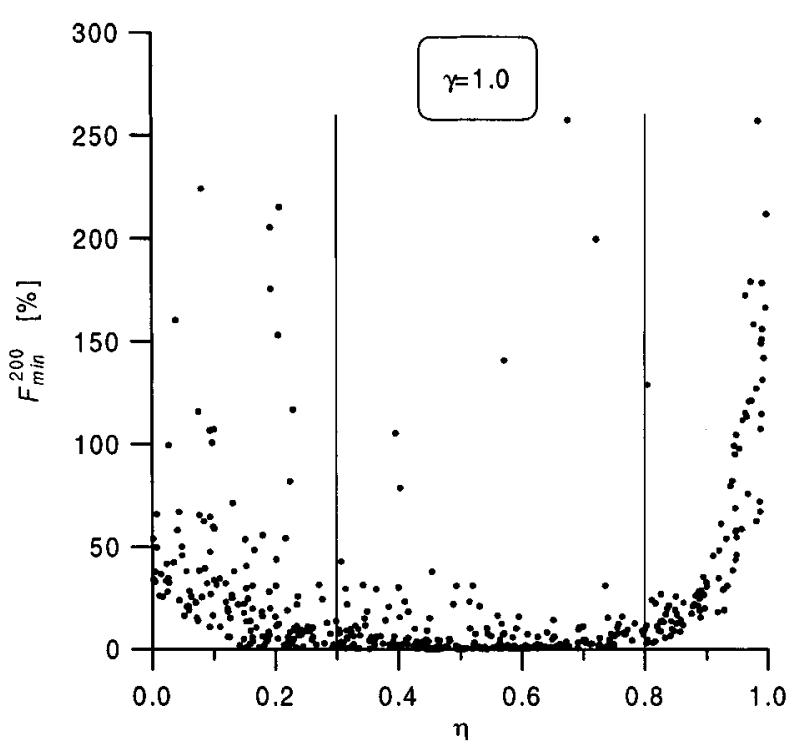

Figure B2. Objective function value vs contraction coefficient $\eta$.

Table B2. A closer analysis of $F_{\min }^{200}$ in the interval $0.3 \leqslant \eta \leqslant 0.8$.

\begin{tabular}{lcccccc}
\hline Case $\backslash \eta$ & 0.3 & 0.4 & 0.5 & 0.6 & 0.7 & 0.8 \\
\hline $\begin{array}{l}\text { Average value } \\
F_{\min }^{200}(\%)\end{array}$ & 13.0 & 10.8 & 7.5 & 7.0 & 10.5 & 13.7 \\
$\begin{array}{l}\text { Std. deviation } \\
F_{\min }^{200}(\%)\end{array}$ & 26.2 & 29.3 & 26.3 & 15.8 & 31.1 & 24.5 \\
$\begin{array}{l}\text { Min. value } \\
F_{\min }^{200}(\%)\end{array}$ & 0.025 & 0.006 & 0.010 & 0.067 & 0.41 & 2.16 \\
$\begin{array}{l}\text { Max. value } \\
F_{\min }^{200}(\%)\end{array}$ & 265.8 & 267.1 & 258.5 & 150.2 & 232.6 & 257.6 \\
\hline
\end{tabular}

It appeared to be favourable to try to restrict the interval for the contraction coefficient. In Figure B2, the values $F_{\text {min }}^{200}$ of the objective function are plotted vs their corresponding contraction coefficients for these optimizations. The interval $0.3 \leqslant \eta \leqslant 0.8$ of the contraction coefficient was regarded to be of most interest and was investigated further by fixing the contraction coefficient stepwise to $\eta=0.3,0.4,0.5,0.6,0.7$, and 0.8 and performing 300 optimizations for each value of $\eta$, with $\zeta$ and $e$ randomly generated as before. Statistical data from this analysis are presented in Table B2. Both the smallest average value and the smallest standard deviation were obtained for $\eta=0.6$ and thus it was natural to fix the contraction coefficient to $\eta=0.6$.

In a final step, the expansion coefficient $\zeta$ was evaluated. As a first attempt, the 300 optimizations with $\gamma=1.0$ and $\eta=0.6$, examined in Table B2, were used in a plot of $F_{\min }^{200}$ vs $\zeta$, see 


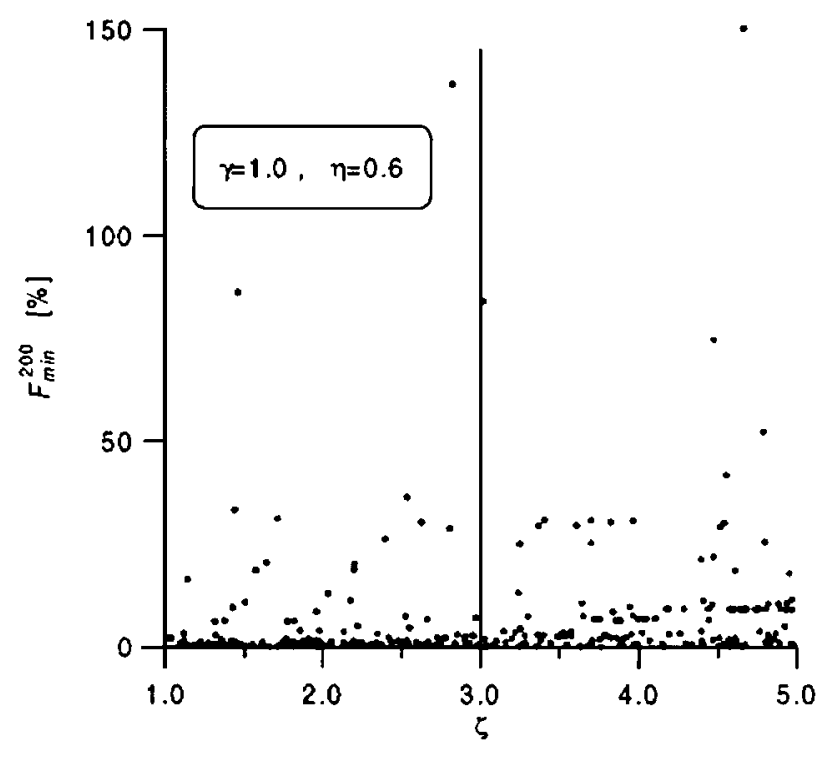

Figure B3. Objective function value vs expansion coefficient $\zeta$.

Table B3. A closer analysis of $F_{\min }^{200}$ in the interval $1.0<\zeta \leqslant 3.0$.

\begin{tabular}{lcccccc}
\hline Case $\backslash \zeta$ & 1.25 & 1.5 & 1.75 & 2.0 & 2.5 & 3.0 \\
\hline $\begin{array}{l}\text { Average value } \\
F_{\min }^{200}(\%)\end{array}$ & 4.5 & 3.5 & 4.6 & 3.7 & 6.3 & 4.9 \\
$\begin{array}{l}\text { Std. deviation } \\
F_{\min }^{200}(\%)\end{array}$ & 12.5 & 10.7 & 20.6 & 8.9 & 22.6 & 15.7 \\
$\begin{array}{l}\text { Min. value } \\
F_{\min }^{200}(\%)\end{array}$ & 0.086 & 0.032 & 0.036 & 0.031 & 0.051 & 0.068 \\
$\begin{array}{l}\text { Max. value } \\
F_{\min }^{200}(\%)\end{array}$ & 199.8 & 108.2 & 246.5 & 88.2 & 204.6 & 220.4 \\
\hline
\end{tabular}

Figure B3. For $\zeta>3.0$, there is a tendency for $F_{\min }^{200}$ to slightly increase with increasing $\zeta$. The interval $1.0<\zeta \leqslant 3.0$ was closer examined by fixing the expansion coefficient in steps to $\zeta=1.25,1.5,1.75,2.0,2.5$ and 3.0 and performing 400 optimizations for each value of $\zeta$, with $e$ randomly generated as before. In Table $\mathrm{B} 3$, statistical data from this analysis are presented. For $\zeta=2.0$, the second smallest average value ( 3.7 per cent compared to 3.5 per cent for $\zeta=1.5$ ) and the smallest standard deviation were obtained. Thus, it was decided to fix the expansion coefficient to $\zeta=2.0$.

It has been noticed that for this evaluation example, the values of the reflection and the contraction coefficients have a much larger influence on the efficiency of the algorithm than the value of the expansion coefficient. A similar observation has also been made by Parkinson and Hutchinson [12]. 


\section{REFERENCES}

1. DeNatale JS. On the calibration of constitutive models by multivariate optimization. Ph.D. Thesis, Department of Civil Engineering, University of California, Davis, 1983.

2. Mattsson H, Axelsson K, Klisinski M. On a constitutive driver as a useful tool in soil plasticity. Advances in Engineering Software 1999; 30:511-528.

3. Runesson K, Axelsson K, Klisinski M. Characteristics of constitutive relations in soil plasticity for undrained behaviour. International Journal of Solids and Structures 1992; 29:363-380.

4. Mattsson H, Axelsson K, Klisinski M. A method to correct yield surface drift in soil plasticity under mixed control and explicit integration. International Journal for Numerical and Analytical Methods in Geomechanics 1997; 21:175-197.

5. Klisinski M. Optimization program for identification of constitutive parameters. Technical Report, Civil Environmental and Architectural Engineering Department, University of Colorado, BO, 1988.

6. Rosenbrock HH. An automatic method for finding the greatest or least value of a function. The Computer Journal 1960; 3:175-184.

7. Nelder JA, Mead R. A simplex method for function minimization. The Computer Journal 1965; 7:308-313.

8. Box MJ. A comparison of several current optimization methods, and the use of transformations in constrained problems. The Computer Journal 1966; 9:67-77.

9. Kincaid D, Cheney W. Numerical Analysis (2nd edn). Brooks/Cole: California, 1996.

10. Rice JR. Experiments on Gram-Schmidt orthogonalization. Mathematics of Computation 1966; 20:325-328.

11. Spendley W, Hext GR, Himsworth FR. Sequential application of simplex designs in optimization and evolutionary operation. Technometrics 1962; 4:441-461.

12. Parkinson JM, Hutchinson D. An investigation into the efficiency of variants on the simplex method. In Numerical Methods for Non-linear Optimization, Lootsma FA (ed.). Academic Press: London, 1972; 115-135.

13. Alawaji H, Runesson K, Sture S, Axelsson K. Implicit integration in soil plasticity under mixed control for drained and undrained response. International Journal for Numerical and Analytical Methods in Geomechanics 1992; 16:737-756.

14. Muir Wood D. Soil Behaviour and Critical State Soil Mechanics. Cambridge University Press: Cambridge, 1990.

15. Wood DM, Wroth CP. Some laboratory experiments related to the results of pressuremeter tests. Geotechnique 1977; 27:181-201.

16. Runesson K, Axelsson K. An initially anisotropic yield criterion for clays. In Proceedings of International Conference on Finite Elements in Nonlinear Solid and Structural Mechanics, Geilo, August 1977, Bergan P et al (eds). Tapir: Trondheim, 1977: 513-533.

17. Yu Y. Testing and modelling of silty and sulphide-rich soils. Ph.D. Thesis 1993:121D, Luleå University of Technology, Luleå, 1993.

18. Wood DM. True triaxial tests on Boston blue clay. In Proceedings of the Tenth International Conference on Soil Mechanics and Foundation Engineering, vol. 1, Stockholm, June 1981. A.A. Balkema: Rotterdam, 1981; 825-830.

19. Fletcher R. Practical Methods of Optimization (2nd edn). Wiley: Chichester, 1987.

20. Sheng, D, Westerberg B, Mattsson H, Axelsson K. Effects of end restraint and strain rate in triaxial tests. Computers and Geotechnics 1997; 21:163-182. 